\title{
Interventions Facilitating Family Communication of Genetic Testing Results and Cascade Screening in Hereditary Breast/Ovarian Cancer or Lynch Syndrome: A Systematic Review and Meta-Analysis
}

\author{
Vasiliki Baroutsou ${ }^{1}$, Meghan L. Underhill-Blazey ${ }^{2}$, Christian Appenzeller-Herzog $^{3}$ and Maria C. Katapodi ${ }^{1, *}$ \\ 1 Department of Clinical Research, Faculty of Medicine, University of Basel, 4055 Basel, Switzerland; \\ vasiliki.baroutsou@unibas.ch \\ 2 School of Nursing, Wilmot Cancer Institute Hereditary Cancer Program, University of Rochester, \\ Rochester, NY 14642, USA; Meghan_Blazey@URMC.Rochester.edu \\ 3 University Medical Library, University of Basel, 4051 Basel, Switzerland; \\ christian.appenzeller-herzog@unibas.ch \\ * Correspondence: maria.katapodi@unibas.ch; Tel.: +41-61-207-04-30
}

check for updates

Citation: Baroutsou, V.;

Underhill-Blazey, M.L.;

Appenzeller-Herzog, C.; Katapodi, M.C. Interventions Facilitating Family Communication of Genetic Testing Results and Cascade Screening in Hereditary Breast/Ovarian Cancer or Lynch Syndrome: A Systematic Review and Meta-Analysis. Cancers 2021, 13, 925. https://doi.org/ 10.3390 /cancers13040925

Academic Editors: Nagrath Deepak and Samuel C. Mok

Received: 31 December 2020

Accepted: 15 February 2021

Published: 23 February 2021

Publisher's Note: MDPI stays neutral with regard to jurisdictional claims in published maps and institutional affiliations.

Copyright: (c) 2021 by the authors. Licensee MDPI, Basel, Switzerland. This article is an open access article distributed under the terms and conditions of the Creative Commons Attribution (CC BY) license (https:/ / creativecommons.org/licenses/by/ $4.0 /)$.
Simple Summary: In general, 5-20\% of all cancers are due to pathogenic variants in cancer genes that are passed down in the family. It is recommended that blood relatives of individuals with such a pathogenic variant have genetic testing, to identify if they also carry the same variant. This information will help their healthcare providers to make individualized cancer screening and prevention plans. However, only around $30 \%$ of at-risk relatives have genetic testing, presumably due to a lack of communication about inherited cancer genes among family members. In this paper, we identified interventions that were designed to improve family communication about hereditary cancer and/or genetic testing among at-risk relatives for two common hereditary cancer syndromes. We analyzed the components of these interventions and synthesized outcomes with statistical methods. Although we identified 14 eligible studies, there are still many unanswered questions about clinical and research implications with diverse samples to be addressed in future studies.

Abstract: Evidence-based guidelines recommend cascade genetic testing of blood relatives of known Hereditary Breast and Ovarian Cancer (HBOC) or Lynch Syndrome (LS) cases, to inform individualized cancer screening and prevention plans. The study identified interventions designed to facilitate family communication of genetic testing results and/or cancer predisposition cascade genetic testing for HBOC and LS. We conducted a systematic review and meta-analysis of randomized trials that assessed intervention efficacy for these two outcomes. Additional outcomes were also recorded and synthesized when possible. Fourteen articles met the inclusion criteria and were included in the narrative synthesis and 13 in the meta-analysis. Lack of participant blinding was the most common risk of bias. Interventions targeted HBOC $(n=5)$; both HBOC and LS $(n=4)$; LS $(n=3)$; or ovarian cancer $(n=2)$. All protocols $(n=14)$ included a psychoeducational and/or counseling component. Additional components were decision aids $(n=4)$, building communication skills $(n=4)$, or motivational interviewing $(n=1)$. The overall effect size for family communication was small $(g=0.085)$ and not significant $(p=0.344)$, while for cascade testing, it was small $(g=0.169)$ but significant $(p=0.014)$. Interventions show promise for improving cancer predisposition cascade genetic testing for HBOC and LS. Future studies should employ family-based approaches and include racially diverse samples.

Keywords: Tier-1 genetic conditions; intervention efficacy; randomized controlled trials; psychoeducational interventions 


\section{Introduction}

Breast, colorectal, ovarian, and endometrial cancers constitute around 30\% of newly diagnosed cancer cases [1,2]. In general, it is considered that approximately $5-10 \%$ of all breast and approximately $20 \%$ of ovarian cancer cases are due to an inherited pathogenic variant associated with Hereditary Breast and Ovarian Cancer (HBOC) syndrome, with some estimates being higher for selected patients and families [3-7]. Lynch Syndrome (LS) accounts for $2-5 \%$ of colorectal and endometrial cancer cases and is associated with increased risk for several other malignancies, including pancreatic, gastric, ovarian, and small bowel cancer [8-10]. Individuals with HBOC or LS are more likely to develop cancer, usually before the age of 50, at which routine cancer screening applies [11].

Germline pathogenic variants associated with HBOC and LS are inherited in an autosomal dominant manner. First- and second-degree relatives and first cousins have $12.5-50 \%$ probability of inheriting the respective cancer predisposition. The availability of cancer genetic services (counselling and testing) for "actionable" hereditary cancer syndromes, such as HBOC and LS, is a significant milestone for effective cancer prevention and control [12,13]. When a pathogenic variant is identified, relatives can be tested with $100 \%$ accuracy. Intensive surveillance starting at a younger age, prophylactic surgery, and chemoprevention can lower the risk of primary and secondary cancers, reducing morbidity and mortality for those who carry the pathogenic variant and medical and insurance costs for those who test negative [14]. The Centers for Disease Control and Prevention (CDC), Office of Public Health Genomics, USA, issued evidence-based recommendations for genetic testing in affected individuals and unaffected relatives when there is a known family history of HBOC, personal history of $B R C A$-related cancers, and LS-related colorectal cancer $[15,16]$. Cascade genetic screening means identifying and testing blood relatives of mutation carriers to determine if they also carry the pathogenic variant and propose risk management options [13].

Despite calls to action for HBOC and LS cascade genetic testing, there are systemic barriers to its implementation. Privacy laws worldwide prohibit healthcare providers from revealing genetic information to anyone except the tested individual. The responsibility to share genetic test results lies almost exclusively with the mutation carrier, who has the right not to disclose this information $[17,18]$. This communication strategy has significant limitations in both ensuring contact with the appropriate people and the transmission of accurate information $[19,20]$. Potential benefits of genetic testing are not being effectively communicated through family networks, leading to more than $50 \%$ of at-risk individuals not using genetic services [21]. Nevertheless, a family-based approach in communicating hereditary cancer risk is advantageous because it is not limited only to those in contact with the healthcare system but may reach relatives through the social functions already existing within the family network [22]. Interventions that support mutation carriers during the disclosure of genetic test results can reduce their psychological distress and provide relatives with accurate and credible information about cascade genetic testing. Technology-enabled education is not inferior to face-to-face genetic consultations [23-25], while it increases access to services and cost-effectiveness [26-28].

In summary, interventions could facilitate communication and access to genetic information and services for families with hereditary predisposition to cancer. The purpose of this study was to identify and synthesize outcomes of psychoeducational interventions designed to facilitate family communication of genetic testing results and/or cancer predisposition cascade genetic testing, with a focus on HBOC and LS. 


\section{Materials and Methods}

\subsection{Literature Extraction}

This systematic review is reported according to PRISMA (Preferred Reporting Items for Systematic Reviews and Meta-Analysis) guidelines [29]. The search strategy was designed to identify available randomized controlled trials (RCTs) that assessed the efficacy of interventions that included family communication of genetic testing results and/or cancer predisposition cascade genetic testing as a primary or a secondary outcome. Several criteria were used to select eligible studies: (1) the intervention had to involve mutation carriers, or blood relatives of known mutation carriers, or individuals with a strong family history indicative of HBOC or LS; (2) the intervention had to include a psychosocial, cognitive, or behavioral component; and (3) participants had to be randomly assigned to either the intervention or the control arm. The search strategies were developed by an information specialist (C.A.-H.) and peer-reviewed by a second information specialist (Dr. Hannah Ewald). The electronic databases Embase via Elsevier, Medline and PsycInfo via Ovid, and the Cochrane Central Register of Controlled Trials (CENTRAL) were searched using text word synonyms and database-specific subject headings for hereditary cancer, genetic counseling/screening, and interventions to promote family communication and/or genetic counseling/screening. For Embase, Medline, and PsycInfo, common RCT filters were applied [30,31] (last search June 15, 2020; Appendix A). References were exported to Endnote X9 [32] and de-duplicated using the Bramer method [33]. Queries were limited to studies published in the English language. Studies published in languages other than English were excluded due to time and resource limitations.

\subsection{Screening, Inclusion, and Exclusion Criteria}

Each research article was screened by title and abstract by at least two members of the research team (VB, MCK, MUB, and Dr. Tarsha Jones), who made an independent assessment among the full-text articles evaluated for eligibility. Disagreements were resolved through consensus. Papers with no original data, such as guidelines, study protocols, and reviews, were excluded. Only original articles assessing family communication and/or cancer predisposition cascade genetic testing for HBOC and LS were included. Studies involving patients with other types of cancer and/or other genetic conditions were excluded to reduce the heterogeneity of the studies analyzed. Full-text analysis was performed on 102 records selected during title and abstract screening.

\subsection{Data Extraction}

Data from eligible articles were extracted and were recorded using Covidence software [34]. We recorded the main author, year of publication, country of origin, study design, demographics of study population, and outcomes. Intervention content and components were also analyzed. In one case, specific intervention characteristics were obtained from an earlier publication that was identified from the reference list of the original article. When authors used more than one instrument to measure the same outcome, extracted data were reported from the most relevant instrument, which was determined by consensus after reviewing wording of each item. A similar procedure was followed when authors reported findings on multiple subscales of instruments, rather than on global scores. The Cochrane Risk of Bias (RoB) tool [35] was used to assess risk of bias in sequence generation (selection bias), allocation concealment (selection bias), blinding of participants and personnel (performance bias), blinding of outcome assessment (detection bias), incomplete outcome data (attrition bias), selective outcome reporting (reporting bias), and other potential sources. Based on the RoB tool, potential sources of bias were characterized as "low", "high", or "unclear" for each included study. Calculation of effect sizes was based on outcome data from the experimental and control arms of each study. 


\subsection{Statistical Analyses}

Outcome data were synthesized using meta-analytic methods [36,37]. The standard mean difference, or the effect size between intervention and control groups, was calculated using Hedges' $g$ unbiased approach, which is similar to Cohen's d statistic [38]. Calculation of effect sizes was based on means, standard deviations, difference in mean scores, odds ratios, $p$-values, and sample sizes of the groups. Data were statistically pooled by the standard meta-analytic approach, meaning that studies were weighted by the inverse of the sampling variance. For studies that did not report the coefficient of correlation $(r)$ between pre- and post- intervention scores, we used Rosenthal's conservative estimate of $r=0.7$ [39]. The random effects model was used as a conservative approach to account for different sources of variation among studies. The $\mathrm{Q}$ statistic was used to assess heterogeneity among studies. A significant $Q$ value indicates lack of homogeneity of findings among studies [36,37]. Due to the small number of studies, we were not able to conduct moderation analyses and examine the effects of intervention characteristics on outcomes. We assessed for publication bias using the Egger's $t$-test with significance values based on one-tailed $p$-values [36,37]. Publication bias can occur because (i) journals are more likely to publish studies with positive results, (ii) authors are less likely to report negative or inconclusive outcomes in multi-outcome studies, or (iii) studies with smaller sample sizes need to detect larger effects to be published than studies with larger samples.

Comprehensive Meta-Analysis V.3○ Software [40] was used for statistical analyses. Reported statistics conform to the PRISMA Statement [29]. Based on conventional standards, effect sizes of $g=0.20,0.50$, and 0.80 were considered small, medium, and large, respectively [38].

\section{Results}

Initial queries identified 2767 articles from all databases and search methods after removing duplicates (see Figure 1 for details). We identified 14 studies that met all inclusion criteria and were included in the narrative synthesis. However, the meta-analysis was based on data extracted from 13 RCTs published between 2002 and July 2019 that assessed family communication and/or cascade genetic testing for HBOC and/or LS among outcomes. Outcomes from one RCT were not included in the calculation of pooled effect sizes due to missing data [41]. Studies measured outcomes at various time points, ranging from one week to 14 months post-intervention. The median time for post-intervention assessments was three months. When studies assessed outcomes multiple times, we used data from the time point closer to three months. For outcomes assessed only once, we used data from that time point. Quality assessment indicated that lack of participant blinding was the most common source of bias among included studies (Table S1). 


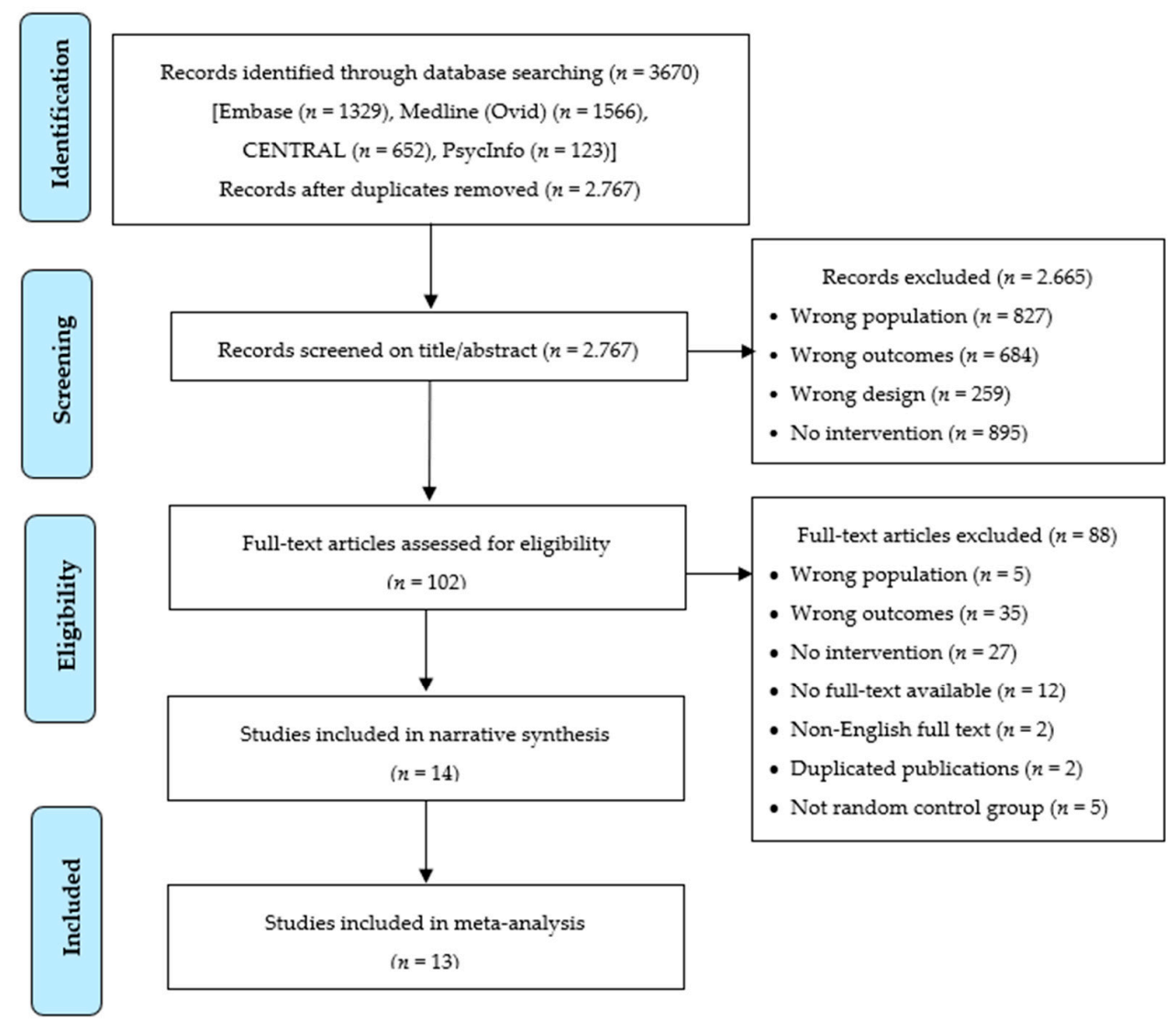

Figure 1. PRISMA flow diagram for study selection.

\subsection{Characteristics and Content of Interventions}

Most interventions targeted HBOC $(n=5)$ [41-45], followed by interventions that targeted multiple hereditary syndromes, including both HBOC and LS $(n=4)$ [46-49], colorectal cancer associated with LS $(n=3)$ [50-52], or ovarian cancer $(n=2)[53,54]$. Characteristics and content of the identified studies are described in Table 1. All protocols $(n=14)$ included a psychoeducational and/or counseling component; $n=5$ included skills building; $n=4$ included a decision aid; and $n=1$ focused on motivational interviewing. The psychoeducational components focused on genetics and hereditary cancer risk, prevention and risk management options, and impact on family and/or family communication. The counseling component was designed to enhance coping, problem solving, self-efficacy, and clarifying personal values. Less often, protocols included resources for participants accessing genetic services $(n=3)$ or additional training and resources for clinicians to enhance referral for genetic counseling $(n=1)$. Most interventions were theoretically driven $(n=9)$; Buckman's six-step strategy for "breaking bad news" was the most frequently mentioned theoretical approach, followed by the Ottawa Decision Support framework. Finally, most studies $(n=9)$ reported various outcomes related to intervention fidelity. Table 1 provides brief details about the content of controls and/or usual care of the identified RCTs. 
Table 1. Characteristics of study interventions.

\begin{tabular}{|c|c|c|c|c|c|c|c|c|c|}
\hline Author/Year & Syndrome/Outcomes * & Intervention & Control & $\begin{array}{l}\text { Theoretical } \\
\text { Framework }\end{array}$ & $\begin{array}{l}\text { Mode of } \\
\text { Delivery }\end{array}$ & Intervener & Dose & Duration & Fidelity \\
\hline $\begin{array}{l}\text { Bodurtha et al., } \\
2014 \text { [46], } \\
\text { KinFact }\end{array}$ & Both/Communication & $\begin{array}{l}\text { Booklet (27-page } \\
\text { personalized information for } \\
\text { family communication about } \\
\text { cancer and cancer genetics) }\end{array}$ & $\begin{array}{l}\text { Pamphlet- } \\
\text { breast, colon } \\
\text { cancer risks, } \\
\text { screening, } \\
\text { services }\end{array}$ & $\begin{array}{l}\text { Health Belief } \\
\text { Model; } \\
\text { Buckman's } \\
\text { 6-step strategy } \\
\text { Breaking Bad } \\
\text { News } \\
\end{array}$ & $\begin{array}{l}\text { Booklet/Pamphlet } \\
\text { Face-to-face } \\
\text { One-on-one }\end{array}$ & $\begin{array}{c}\text { Trained } \\
\text { Personnel }\end{array}$ & Once & 20-min & $\mathrm{NR} \sim$ \\
\hline $\begin{array}{c}\text { Dekker et al., } \\
2015 \text { [50] }\end{array}$ & $\begin{array}{c}\text { CRC } \\
* * / \text { Communication } \\
\text { Cascade testing } \\
\text { Knowledge }\end{array}$ & $\begin{array}{c}\text { Website (CRC risk, risk } \\
\text { calculators, decision aid) }+ \\
\text { Brochure (familial CRC risk, } \\
\text { prevention) }+30-\text { min } \\
\text { Clinician education }+ \\
\text { Referral cards (criteria) }\end{array}$ & Usual care & NR & $\begin{array}{l}\text { Website + } \\
\text { Brochure }\end{array}$ & $\begin{array}{c}\text { Self- } \\
\text { administered }\end{array}$ & NR & NR & $\begin{array}{l}67 \% \text { used } \\
\text { website }\end{array}$ \\
\hline $\begin{array}{c}\text { Eijzenga et al., } \\
2018 \text { [47] }\end{array}$ & $\begin{array}{c}\text { Both/Communication } \\
\text { Knowledge Perceived } \\
\text { risk }\end{array}$ & $\begin{array}{c}\text { Standard genetic counseling } \\
\text { + Phone call-motivational } \\
\text { interviewing (enhance family } \\
\text { communication, knowledge, } \\
\text { motivation, self-efficacy, } \\
\text { solutions) }\end{array}$ & $\begin{array}{l}\text { Standard } \\
\text { genetic } \\
\text { counseling }\end{array}$ & $\begin{array}{l}\text { Motivational } \\
\text { interviewing }\end{array}$ & Telephone & $\begin{array}{l}\text { Psychosocial } \\
\text { Worker }\end{array}$ & Once & NR & $\begin{array}{c}\text { 33\% random } \\
\text { check } \\
\text { interview } \\
\text { recording }\end{array}$ \\
\hline $\begin{array}{l}\text { Hodgson et al., } \\
2016[48]\end{array}$ & $\begin{array}{l}\text { Multiple incl. HBOC + } \\
\text { LSCascade testing }\end{array}$ & $\begin{array}{l}\text { Enhanced genetic counseling } \\
\text { over telephone with } \\
\text { emphasis on family } \\
\text { communication + Pedigree }\end{array}$ & Pedigree & NR & $\begin{array}{l}\text { Telephone } \\
\text { One-on-one }\end{array}$ & $\begin{array}{l}\text { Genetic } \\
\text { Coun- } \\
\text { selors }\end{array}$ & 2-3 times & $\begin{array}{c}12 \\
\text { months }\end{array}$ & NR \\
\hline $\begin{array}{l}\text { Katapodi et al., } \\
2018 \text { [42] Family } \\
\text { Gene Toolkit }\end{array}$ & $\begin{array}{l}\text { HBOC / Communica- } \\
\text { tionKnowledge } \\
\text { Perceived risk }\end{array}$ & $\begin{array}{l}\text { Webinar (power point, live } \\
\text { presentations about cancer } \\
\text { genetics, risk, genetic } \\
\text { counseling, coping, family } \\
\text { communication) + Decision } \\
\text { aid + Communication skills } \\
\text { building + Phone call }\end{array}$ & $\begin{array}{l}\text { Wait-listed } \\
\text { control }\end{array}$ & $\begin{array}{c}\text { Theory of Stress } \\
\text { and Coping }\end{array}$ & $\begin{array}{l}\text { Web-based + } \\
\text { Telephone } \\
\text { Face-to-face } \\
\text { One-on-family + } \\
\text { One-on-one }\end{array}$ & $\begin{array}{l}\text { Genetic } \\
\text { Counselor } \\
\text { + Master's } \\
\text { Oncology } \\
\text { Nurse }\end{array}$ & $\begin{array}{l}2 \text { webinars } \\
45-60 \text { min } \\
\text { per } \\
\text { webinar }+ \\
20 \text { min } \\
\text { phone call }\end{array}$ & $\begin{array}{l}3 \text { weeks } \\
110-140 \\
\text { min }\end{array}$ & $\begin{array}{l}71 \% \\
\text { completion } \\
\text { rate }\end{array}$ \\
\hline
\end{tabular}


Table 1. Cont.

\begin{tabular}{|c|c|c|c|c|c|c|c|c|c|}
\hline Author/Year & Syndrome/Outcomes * & Intervention & Control & $\begin{array}{l}\text { Theoretical } \\
\text { Framework }\end{array}$ & $\begin{array}{l}\text { Mode of } \\
\text { Delivery }\end{array}$ & Intervener & Dose & Duration & Fidelity \\
\hline $\begin{array}{l}\text { Loader et al., } \\
2002[51]\end{array}$ & CRC/Cascade testing & $\begin{array}{l}\text { Brochure (hereditary cancer, } \\
\text { risk factors, prevention, } \\
\text { genetic testing, family } \\
\text { communication) + Invitation } \\
\text { to counseling + Letter genetic } \\
\text { counseling }\end{array}$ & $\begin{array}{c}\text { Physician } \\
\text { education (CRC } \\
\text { risk, } \\
\text { information } \\
\text { about referrals } \\
\text { to counseling) }\end{array}$ & NR & $\begin{array}{c}\text { Brochure + } \\
\text { Letter } \\
\text { Face-to-face } \\
\text { One-on-one }\end{array}$ & $\begin{array}{l}\text { Mail, Self- } \\
\text { administered }\end{array}$ & Once & NR & $47 \%$ counseled \\
\hline $\begin{array}{c}\text { Lobb et al., } 2002 \\
{[43]}\end{array}$ & $\begin{array}{l}\text { HBOC/Anxiety } \\
\text { Depression }\end{array}$ & $\begin{array}{l}\text { Audio-recording of genetic } \\
\text { consultation }\end{array}$ & Usual care & NR & Audiotapes & $\begin{array}{c}\text { Self- } \\
\text { administered }\end{array}$ & NR & NR & $\begin{array}{l}51 \% \text { listened } \\
\text { tape once }\end{array}$ \\
\hline $\begin{array}{c}\text { McInerney-Leo } \\
\text { et al., } 2004^{* * *} \\
{[41]}\end{array}$ & HBOC & $\begin{array}{c}\text { Family education + Problem } \\
\text { Solving Training } \\
\text { (expectations, concerns, } \\
\text { feelings) for task- and } \\
\text { emotional-focused coping } \\
\text { and problem solving + } \\
\text { Telephone interview }\end{array}$ & $\begin{array}{c}\text { Family } \\
\text { education }+ \\
\text { Client-centered } \\
\text { counseling }+ \\
\text { Telephone } \\
\text { interview }\end{array}$ & $\begin{array}{l}\text { Cognitive- } \\
\text { Behavioral } \\
\text { Theory }\end{array}$ & $\begin{array}{c}\text { Face-to-face or } \\
\text { TelephoneOne- } \\
\text { on-family }+ \\
\text { One-on-one }\end{array}$ & $\begin{array}{l}\text { Trained } \\
\text { Provider }\end{array}$ & Once & $60 \mathrm{~min}$ & $\begin{array}{l}\text { Standardized } \\
\text { protocol }\end{array}$ \\
\hline $\begin{array}{l}\text { Montgomery } \\
\text { et al., } 2013 \text { [44] }\end{array}$ & $\begin{array}{l}\text { HBOC/Communication } \\
\text { Depression }\end{array}$ & $\begin{array}{c}\text { Counseling (risk factors, } \\
\text { personal risk, pedigree) + } \\
\text { Communication skills } \\
\text { building (who, how, extent } \\
\text { willing to know, share results, } \\
\text { emotional responses, } \\
\text { resources) }\end{array}$ & $\begin{array}{l}\text { Wellness } \\
\text { education } \\
\text { (nutrition, } \\
\text { exercise) + List } \\
\text { of nutrition } \\
\text { websites }\end{array}$ & $\begin{array}{l}\text { Buckman's } \\
\text { 6-step strategy } \\
\text { Breaking Bad } \\
\text { News + Theory } \\
\text { of Planned } \\
\text { Behavior }\end{array}$ & $\begin{array}{l}\text { Face-to-face } \\
\text { One-on-one }\end{array}$ & $\begin{array}{c}\text { Genetic } \\
\text { Counselor } \\
+ \text { Research } \\
\text { Staff }\end{array}$ & NR & NR & NR \\
\hline $\begin{array}{c}\text { Niu et al., } 2019 \\
\text { [52] }\end{array}$ & $\begin{array}{l}\text { CRC/Communication } \\
\text { Anxiety Depression }\end{array}$ & $\begin{array}{c}\text { Genetic counseling }+ \text { Clinical } \\
\text { exome sequencing }(21 \text { to }>50 \\
\text { actionable genes) }+ \\
\text { Additional genetic } \\
\text { information }\end{array}$ & $\begin{array}{c}\text { Counseling + } \\
\text { Tumor testing } \\
\text { OR panel testing } \\
+ \text { Review family } \\
\text { history }\end{array}$ & NR & $\begin{array}{c}\text { Telephone or } \\
\text { Face-to- } \\
\text { faceOne-on-one }\end{array}$ & $\begin{array}{c}\text { Genetic } \\
\text { Counselor } \\
\text { or } \\
\text { Geneticist }\end{array}$ & NR & NR & NR \\
\hline
\end{tabular}


Table 1. Cont.

\begin{tabular}{|c|c|c|c|c|c|c|c|c|c|}
\hline Author/Year & Syndrome/Outcomes * & Intervention & Control & $\begin{array}{l}\text { Theoretical } \\
\text { Framework }\end{array}$ & $\begin{array}{l}\text { Mode of } \\
\text { Delivery }\end{array}$ & Intervener & Dose & Duration & Fidelity \\
\hline $\begin{array}{l}\text { Roshanai et al., } \\
2009 \text { [49] }\end{array}$ & $\begin{array}{c}\text { Both/Communication } \\
\text { Knowledge Anxiety } \\
\text { DepressionPerceived } \\
\text { risk }\end{array}$ & $\begin{array}{l}\text { Genetic counseling + } \\
\text { Extended meeting nurse } \\
\text { specialist (pedigree, cancer } \\
\text { risk, 6-step strategy for } \\
\text { family communication) + } \\
\text { Pamphlet + Videotape of } \\
\text { counseling + Copies } \\
\text { pedigree, medical records }\end{array}$ & $\begin{array}{c}\text { Genetic } \\
\text { counseling + } \\
\text { Short meeting } \\
\text { nurse specialist } \\
\text { (intention } \\
\text { inform relatives) } \\
+ \text { Videotape of } \\
\text { counseling }\end{array}$ & $\begin{array}{c}\text { Buckman's } \\
\text { 6-step strategy } \\
\text { Breaking Bad } \\
\text { News }\end{array}$ & $\begin{array}{c}\text { Clinical } \\
\text { settingFace-to- } \\
\text { face } \\
\text { One-on-one }\end{array}$ & $\begin{array}{l}\text { Genetic } \\
\text { Counselor } \\
+ \text { Nurse } \\
\text { Specialist }\end{array}$ & Once & NR & $\begin{array}{l}\text { 19-item survey } \\
\text { counselees }\end{array}$ \\
\hline $\begin{array}{c}\text { Tiller et al., } 2006 \\
\text { [53] }\end{array}$ & $\begin{array}{c}\text { Ovarian } \\
\text { Cancer/Knowledge } \\
\text { Anxiety }\end{array}$ & $\begin{array}{l}\text { Decision aid (booklet on risk } \\
\text { factors, family history and } \\
\text { risk, genetic testing, } \\
\text { prevention) + Values } \\
\text { clarification }\end{array}$ & $\begin{array}{l}\text { General } \\
\text { education } \\
\text { pamphlet }\end{array}$ & $\begin{array}{c}\text { Ottawa Decision } \\
\text { Support } \\
\text { Framework }\end{array}$ & Pamphlet & $\begin{array}{c}\text { Self- } \\
\text { administered }\end{array}$ & Once & NR & $\begin{array}{l}88 \% \text { review } \\
\text { booklet }\end{array}$ \\
\hline $\begin{array}{l}\text { Vogel et al., } 2019 \\
\text { [54] mAGIC }\end{array}$ & $\begin{array}{l}\text { Ovarian can- } \\
\text { cer/Communication } \\
\text { Cascade testing } \\
\text { Knowledge }\end{array}$ & $\begin{array}{l}\text { Mobile app tailored messages } \\
\text { (motivation, positive } \\
\text { feedback, triggers) + Videos } \\
\text { (genetic counseling, testing, } \\
\text { personal health, cancer } \\
\text { genetics, self-care, } \\
\text { self-efficacy) + Training how } \\
\text { to use mAGIC + Pamphlet } \\
\text { (ovarian cancer risk, } \\
\text { counseling, services) }\end{array}$ & $\begin{array}{l}\text { Usual care + } \\
\text { Pamphlet } \\
\text { (hereditary } \\
\text { cancer risk, } \\
\text { counseling, } \\
\text { services) }\end{array}$ & $\begin{array}{l}\text { Health Belief } \\
\text { Model + Fogg } \\
\text { Behavioral } \\
\text { Model of Mobile } \\
\text { Persuasion }\end{array}$ & $\begin{array}{l}\text { Mobile app + } \\
\text { Pamphlet }\end{array}$ & $\begin{array}{c}\text { Self- } \\
\text { administered }\end{array}$ & $\begin{array}{l}\text { Once per } \\
\text { day10-15 } \\
\text { min per } \\
\text { day }\end{array}$ & $\begin{array}{l}7 \text { days70- } \\
90 \\
\min \end{array}$ & NR \\
\hline $\begin{array}{l}\text { Wakefield et al., } \\
2008 \text { [45] }\end{array}$ & $\begin{array}{c}\text { HBOC/Cascade testing } \\
\text { Knowledge }\end{array}$ & $\begin{array}{c}\text { Decision aid (40-page } \\
\text { booklet, hereditary cancer, } \\
\text { testing, impact on individual } \\
\text { and family) + Values } \\
\text { clarification }\end{array}$ & $\begin{array}{c}\text { Pamphlet } \\
\text { (4-page } \\
\text { education about } \\
\text { HBOC genetic } \\
\text { testing) }\end{array}$ & $\begin{array}{c}\text { Ottawa Decision } \\
\text { Support } \\
\text { Framework }\end{array}$ & Brochure/Pamphle & $\begin{array}{l}\text { Self- } \\
\text { et } \\
\text { administered }\end{array}$ & NR & NR & $\begin{array}{l}\quad 70 \% \\
\text { intervention } \\
\text { read booklet }\end{array}$ \\
\hline
\end{tabular}

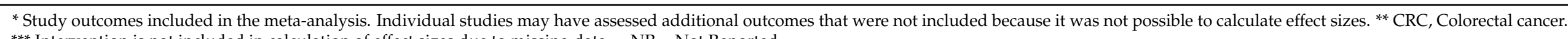

$* * *$ Intervention is not included in calculation of effect sizes due to missing data. $\sim$ NR $=$ Not Reported. 


\subsection{Intervention Mode of Delivery and Intervener}

Few protocols $(n=2)$ targeted implicitly or explicitly more than one member from the same family. Most interventions $(n=8)$ required extensive counseling sessions with a healthcare provider, often specified as a genetic counselor/geneticist, Master's-prepared nurse, or psychosocial worker. Counseling involved mostly one-on-one sessions and was delivered entirely or partially over the telephone $(n=7)$. Interventions were developed either exclusively as booklets $(n=3)$ or included a paper handout as a complementary component $(n=4)$. Technology-enabled interventions were delivered either via the World Wide Web $(n=2)$, as a mobile app $(n=1)$, or included the audio recordings of the counseling sessions $(n=1)$.

\subsection{Intervention Dose and Duration}

The dose and duration of "received intervention" was not consistently reported among studies. Most protocols $(n=9)$ specified a dose of intervention that ranged from 1 to 7 contacts with participants, with an overall duration ranging from 20 to $140 \mathrm{~min}$, over 7 days, 3 weeks, or 12 months.

\subsection{Characteristics of Samples}

Most studies were conducted in the US $(n=7)$, followed by Australia $(n=4)$, the Netherlands $(n=2)$, and Sweden $(n=1)$. Table 2 summarizes the sample characteristics of the 14 interventions included in the narrative synthesis. Sample sizes ranged from 24 to 490 , with a total of 2968 participants across all studies. Recruitment in most studies $(n=8)$ was from outpatient settings. Enrollment rates varied from $23 \%$ to $96 \%$ of those approached, with an average enrollment of $71 \%$ across studies. Attrition ranged from $13 \%$ to $59 \%$, with an average attrition of $27 \%$ across studies. Reasons for attrition were not consistently reported.

Most studies $(n=10)$ included over $50 \%$ female participants, the majority including $100 \%$ females $(n=7)$; few focused exclusively on ovarian cancer $(n=2)$; the remaining focused on HBOC $(n=5)$. A larger proportion of males was included in studies focusing on colorectal cancer and only one included a majority of male participants [50]. Race was not consistently reported, especially for studies conducted outside the US $(n=7)$. Studies that reported participants' race included only or primarily White individuals, and only one included 59\% Black individuals [46]. The reported mean ages ranged from 33 to 61 . Participants were mostly well-educated among the studies that reported educational level $(n=7)$.

Most studies ( $n=11)$ included both affected and unaffected individuals. Four studies reported whether participants had a pathogenic variant associated with cancer; all others focused on personal and/or family history of cancer to describe risk. 
Table 2. Sample characteristics.

\begin{tabular}{|c|c|c|c|c|c|c|c|c|c|c|}
\hline $\begin{array}{c}\text { Author/Year } \\
\text { Country }\end{array}$ & Setting & $\begin{array}{c}\text { Sample } \\
\mathbf{N}\end{array}$ & $\begin{array}{c}\text { Cancer } \\
\text { Type/Stage/PDx * }\end{array}$ & $\begin{array}{c}\text { Carrier of PV } \\
\text { FH } \\
\text { *** or }\end{array}$ & $\begin{array}{c}\text { Age Mean } \pm \text { SD or } \\
\text { Range }\end{array}$ & Sex & Race & $\begin{array}{c}\text { Education } \% \\
\leq \mathrm{HS}^{\wedge}\end{array}$ & Enrollment & Attrition \\
\hline $\begin{array}{c}\text { Bodurtha et., } \\
\text { al } 2014 \text { [46], } \\
\text { USA }\end{array}$ & Outpatient & 490 & $\begin{array}{l}\text { Stage/type NR: } \\
\text { HBOC or CRC risk }\end{array}$ & $\begin{array}{l}75 \% \mathrm{FDR}^{+} \text {any } \\
\text { cancer } \\
10 \% \text { FH breast or } \\
\text { CRC }\end{array}$ & $33.4 \pm 11.9$ & $100 \%$ female & $\begin{array}{c}59 \% \text { Black } \\
33 \% \text { White } \\
8 \% \\
\text { Other/Multiple }\end{array}$ & $\begin{array}{c}41 \% \\
16 \% \text { missing }\end{array}$ & $61 \%$ & $42 \%$ \\
\hline $\begin{array}{c}\text { Dekker et al., } \\
2015 \text { [50], } \\
\text { Netherlands }\end{array}$ & Hospital & 384 & $\begin{array}{c}100 \% \text { CRC } \\
\text { I: } 86.4 \% \text { Stage I-III } \\
\text { C: } 86.55 \text { Stage I-III }\end{array}$ & $\begin{array}{l}\text { I: } 9 \% \text { high risk } \\
\text { C:13\% high risk }\end{array}$ & $\begin{array}{l}\text { I: } 60 \pm 8.2 \\
\text { C: } 59 \pm 7.5\end{array}$ & $\begin{array}{l}\text { I: } 71 \% \text { male } \\
\text { C: } 66 \% \text { male }\end{array}$ & $\mathrm{NR} \sim$ & NR & $55 \%$ & $59 \%$ \\
\hline $\begin{array}{c}\text { Eijzenga et al., } \\
2018 \text { [47], } \\
\text { Netherlands }\end{array}$ & Hospital & 305 & $\begin{array}{c}\text { Stage/type NR; } \\
\text { HBOC or CRC risk } \\
\text { I: } 70 \% \text { PDx } \\
\text { C: } 73 \% \text { PDx }\end{array}$ & $\begin{array}{l}\text { I: } 9 \% \text { PV } \\
\text { C: } 12 \% \text { PV }\end{array}$ & $\begin{array}{l}\mathrm{I}: 53.1 \pm 10.1 \\
\mathrm{C}: 54.4 \pm 12.4\end{array}$ & $\begin{array}{l}\text { I: } 75 \% \text { female } \\
\text { C: } 75 \% \text { female }\end{array}$ & NR & I: $36 \% C: 30 \%$ & $90 \%$ & $21 \%$ \\
\hline $\begin{array}{l}\text { Hodgson et al., } \\
\text { 2016 [48], } \\
\text { Australia }\end{array}$ & $\begin{array}{l}\text { Hospital and } \\
\text { Genetic Clinic }\end{array}$ & 95 & $\begin{array}{l}\text { Stage/type NR; } \\
\text { HBOC and LS }\end{array}$ & $\begin{array}{l}\text { I: } 57.8 \% \text { "actionable" } \\
\text { groupC: } 50.0 \% \\
\text { "actionable" group }\end{array}$ & $\begin{array}{l}\text { I: } 49.5 \pm 14.9 \\
\text { C: } 45.8 \pm 13.9\end{array}$ & $\begin{array}{l}\text { I: } 50 \% \text { female } \\
\text { C: } 48 \% \text { female }\end{array}$ & NR & NR & $57 \%$ & $53 \%$ \\
\hline $\begin{array}{l}\text { Katapodi et al., } \\
2018 \text { [42], USA }\end{array}$ & Outpatient & 24 & $\begin{array}{c}\text { Stage/type NR: } \\
\text { HBOC } \\
\text { 40\% PDx Breast } \\
\text { 10\% PDx Ovarian } \\
\text { 20\% PDx Other }\end{array}$ & $12 \mathrm{PV}$ & $41 \pm 13$ & $100 \%$ female & $100 \%$ White & NR & $23 \%$ & $29 \%$ \\
\hline $\begin{array}{l}\text { Loader et al., } \\
2002 \text { [51], USA }\end{array}$ & $\begin{array}{l}\text { Cancer } \\
\text { Registry }\end{array}$ & 101 & $\begin{array}{l}100 \% \text { PDxCRC; } \\
\text { stage NR }\end{array}$ & $\begin{array}{c}100 \% \geq 1 \mathrm{FDR} \text { or } \\
\mathrm{SDR}^{++} \mathrm{CRC}\end{array}$ & $\begin{array}{c}\text { Not Counseled: } 57.3 \\
\pm 6.9 \\
\text { Counseled:59.2 } \\
6.5\end{array}$ & $53 \%$ female & 93\% White & NR & $71 \%$ & $13 \%$ \\
\hline $\begin{array}{l}\text { Lobb et al., } \\
2002 \text { [43], } \\
\text { Australia }\end{array}$ & Outpatient & 193 & $\begin{array}{c}\text { Stage/type NR; } \\
\text { HBOC } \\
\text { I: } 42 \% \text { PDx } \\
\text { C: } 45 \% \text { PDx }\end{array}$ & NR & $\begin{array}{l}\text { I: } 45 \\
\text { C: } 44\end{array}$ & $100 \%$ female & NR & $\begin{array}{l}\text { I: } 47 \% \\
\text { C: } 50 \%\end{array}$ & $88 \%$ & $18 \%$ \\
\hline $\begin{array}{l}\text { McInerney- } \\
\text { Leo et al., } 2004 \\
\text { [41], USA }\end{array}$ & NR & 262 & $\begin{array}{l}\text { Stage/type NR; } \\
\text { HBOC families }\end{array}$ & $\begin{array}{c}26 \% \text { PV } \\
85 \% \text { genetic testing }\end{array}$ & $55 \% \geq 40$ & $65 \%$ female & Mostly White & NR & $47 \%$ & $19 \%$ \\
\hline
\end{tabular}


Table 2. Cont.

\begin{tabular}{|c|c|c|c|c|c|c|c|c|c|c|}
\hline $\begin{array}{l}\text { Author/Year } \\
\text { Country }\end{array}$ & Setting & $\begin{array}{c}\text { Sample } \\
\text { N }\end{array}$ & $\begin{array}{c}\text { Cancer } \\
\text { Type/Stage/PDx * }\end{array}$ & $\begin{array}{c}\text { Carrier of PV } \\
\text { FH } \\
* * *\end{array}$ & $\begin{array}{c}\text { Age Mean } \pm \text { SD or } \\
\text { Range }\end{array}$ & Sex & Race & $\begin{array}{c}\text { Education } \% \\
\leq \text { HS }^{\wedge}\end{array}$ & Enrollment & Attrition \\
\hline $\begin{array}{l}\text { Montgomery } \\
\text { et al., } 2013 \\
\text { [44], USA }\end{array}$ & Outpatient & 422 & $\begin{array}{c}\text { Stage/type NR; } \\
\text { HBOC }\end{array}$ & NR & $48.5 \pm 11.0$ & $100 \%$ female & 95\% White & $77 \%$ & $96 \%$ & $41 \%$ \\
\hline $\begin{array}{c}\text { Niu et al., } 2019 \\
\text { [52], USA }\end{array}$ & Outpatient & 190 & $\begin{array}{l}\text { I: } 33.68 \% \text { CRC PDx } \\
\text { C: } 36.84 \% \text { CRC PDx }\end{array}$ & NR & $\begin{array}{l}\text { I: } 53.4 \pm 12.5 \\
\text { C: } 51.8 \pm 14.0\end{array}$ & $\begin{array}{l}\text { I: } 46 \% \text { female } \\
\text { C: } 57 \% \text { female }\end{array}$ & $\begin{array}{l}\text { I: } 81 \% \text { White } \\
\text { C: } 84 \% \text { White }\end{array}$ & NR & NR & $26 \%$ \\
\hline $\begin{array}{l}\text { Roshanai et al., } \\
\text { 2009 [49], } \\
\text { Sweden }\end{array}$ & Outpatient & 147 & $\begin{array}{l}\text { HBOC, CRC riskI: } \\
\text { 38.36\% PDxC: } \\
\text { 35.14\% PDx }\end{array}$ & $\begin{array}{c}\text { I: } 77 \% \text { No PDx } \\
>20 \% \text { risk } \\
79 \% \text { PDx }>20 \% \text { risk } \\
\text { C: } 81 \% \text { No PDx } \\
>20 \% \text { risk } \\
70 \% \text { PDx }>20 \% \text { risk }\end{array}$ & $56(23-84)$ & $\begin{array}{l}\text { I: } 92 \% \text { female } \\
\text { C: } 89 \% \text { female }\end{array}$ & NR & NR & $66 \%$ & $15 \%$ \\
\hline $\begin{array}{l}\text { Tiller et al., } \\
2006 \text { [53], } \\
\text { Australia }\end{array}$ & Outpatient & 131 & $\begin{array}{l}\text { Ovarian cancer } \\
\text { I: } 51.5 \% \text { PDx } \\
\text { C: } 52.4 \% \text { PDx }\end{array}$ & $\begin{array}{l}\text { I: } 74.2 \% \mathrm{FH} \\
\mathrm{C}: 71.4 \% \mathrm{FH}\end{array}$ & $\begin{array}{l}\text { I: } 45.8 \\
\text { C: } 46.3\end{array}$ & $100 \%$ female & NR & $\begin{array}{l}\text { I: } 29 \% \\
\text { C: } 29 \%\end{array}$ & $92 \%$ & $17 \%$ \\
\hline $\begin{array}{l}\text { Vogel et al., } \\
2019 \text { [54], USA }\end{array}$ & Outpatient & 104 & $\begin{array}{c}\text { Ovarian cancer } \\
100 \% \text { PDx } \\
\text { I: } \geq 74 \% \text { Stage III } \\
\text { C: } \geq 75 \% \text { Stage III }\end{array}$ & NR & $\begin{array}{c}\mathrm{I}: 60.9 \pm 10.7 \\
\mathrm{C}: 61 \pm 12\end{array}$ & $100 \%$ female & $\begin{array}{l}\text { I: } 91 \% \text { White } \\
\text { C: } 88 \% \text { White }\end{array}$ & $\begin{array}{l}\text { I: } 20.8 \% \\
\text { C: } 18 \%\end{array}$ & $82 \%$ & $13 \%$ \\
\hline $\begin{array}{l}\text { Wakefield et., } \\
\text { al } 2008 \text { [45], } \\
\text { Australia }\end{array}$ & Outpatient & 120 & $\begin{array}{c}\text { Type NR; } \\
\text { HBOC } \\
\text { I:56.1\% PDx } \\
\text { C:65.1\% PDx }\end{array}$ & $\begin{array}{c}100 \% \mathrm{FH} \\
\text { HBOC—cancer }\end{array}$ & $\begin{array}{l}\text { I: } 45.8(21-73) \\
\text { C: } 49.6(22-75)\end{array}$ & $100 \%$ female & NR & $\begin{array}{l}\text { I: } 26.3 \% \\
\text { C: } 36.5 \%\end{array}$ & $94 \%$ & $17 \%$ \\
\hline
\end{tabular}

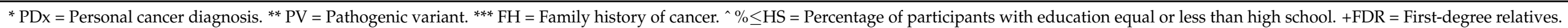
$++\mathrm{SDR}=$ Second-degree relatives. $\sim \mathrm{NR}=$ Not Reported. 


\subsection{Effect Sizes Obtained for Outcomes}

Table 3 presents an overview of meta-analytic findings for outcomes assessed. Family communication was the most commonly assessed outcome $(n=8)$, followed by knowledge $(n=7)$, cascade genetic testing $(n=6)$, anxiety $(n=4)$, depression $(n=4)$, and perceived risk $(n=3)$. Primary studies assessed additional outcomes, i.e., decisional conflict, decisional regret, coping, distress, and self-efficacy. However, calculation of pooled effect sizes for these additional outcomes was not possible, either because there were less than three studies or due to missing data. The table provides the pooled effect sizes for assessed outcomes, $95 \%$ confidence intervals, assessment of heterogeneity across studies ( $Q$ statistic), and Egger's $t$-test for publication bias. Forest plots for each outcome are shown. Forest plots depict the effect sizes calculated for each study by outcome ( $\mathbf{\square}$ symbol) as well as the overall effect size obtained for the outcome across studies ( symbol). Forest plots also indicate whether effects obtained in each study and across studies favor the control or the intervention.

Table 3. Pooled effect sizes of outcomes.

\begin{tabular}{cccccc}
\hline Outcomes & $\begin{array}{c}\text { Number of } \\
\text { Trials }\end{array}$ & Overall Sample N & $\begin{array}{c}\text { Pooled Effect Size } \\
\text { Hedges' } \mathbf{g}(\mathbf{9 5 \%} \text { CI) }\end{array}$ & $\begin{array}{c}\text { Q for } \\
\text { Heterogeneity }\end{array}$ & $\begin{array}{c}\text { Egger's } t \text {-Test for } \\
\text { Publication Bias }\end{array}$ \\
\hline Family communication & 8 & 2066 & $0.085(-0.091-0.261)$ & $15.50^{*}$ & 0.53 \\
Cascade genetic testing & 4 & 703 & $0.169(0.034-0.305)^{*}$ & 0.93 & -0.66 \\
Knowledge & 7 & 1215 & $0.244(0.109-0.379)^{*}$ & $15.10^{*}$ & 0.50 \\
Anxiety & 4 & 661 & $0.033(-0.132-0.198)$ & 6.14 & $-4.17^{*}$ \\
Depression & 4 & 952 & $0.183(0.033-0.334)^{*}$ & 2.39 & 2.89 \\
Risk perception & 3 & 476 & $0.007(-0.230-0.250)$ & 1.69 & 0.97 \\
\hline
\end{tabular}

${ }^{*} p$-value $\leq 0.05$.

Family communication was conceptualized by primary studies most commonly as the number of relatives contacted/informed about the pathogenic variant, as well as frequency of contact and openness/ease of family communication. The $Q$ statistic indicates significant heterogeneity among the eight studies that evaluated changes in family communication. The overall effect size was small and not significant, $g=0.085(p=0.344)$. (Figure 2). Among the eight studies, three assessed family communication as a secondary outcome [50,52,54]; removing these studies did not change the significance of the pooled effect size.

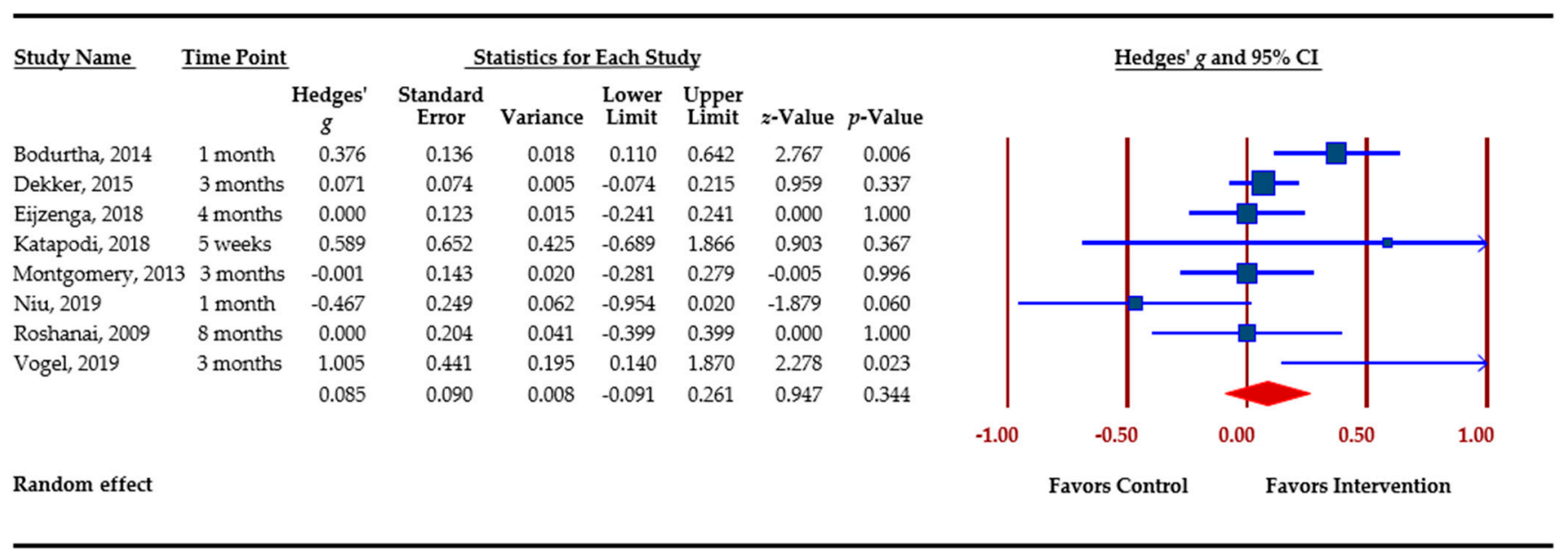

Figure 2. Family communication.

Cascade genetic testing was assessed by six primary studies as uptake of genetic testing by relatives and/or contact with genetic services and request for genetic consultation. The assessment was based on participants' self-reports, and/or less often on clinic records. The overall effect size was small and not significant, $g=0.086(-0.075-0.247)(p=0.295)$. 
However, two of these studies $[51,53]$ assessed cascade genetic testing as a secondary outcome. Removing these two studies changed the overall effect size, which remained small but significant, $g=0.169(p=0.014)$. (Figure 3$)$. Effect sizes among primary studies ranged from 0.010 to 0.368 .

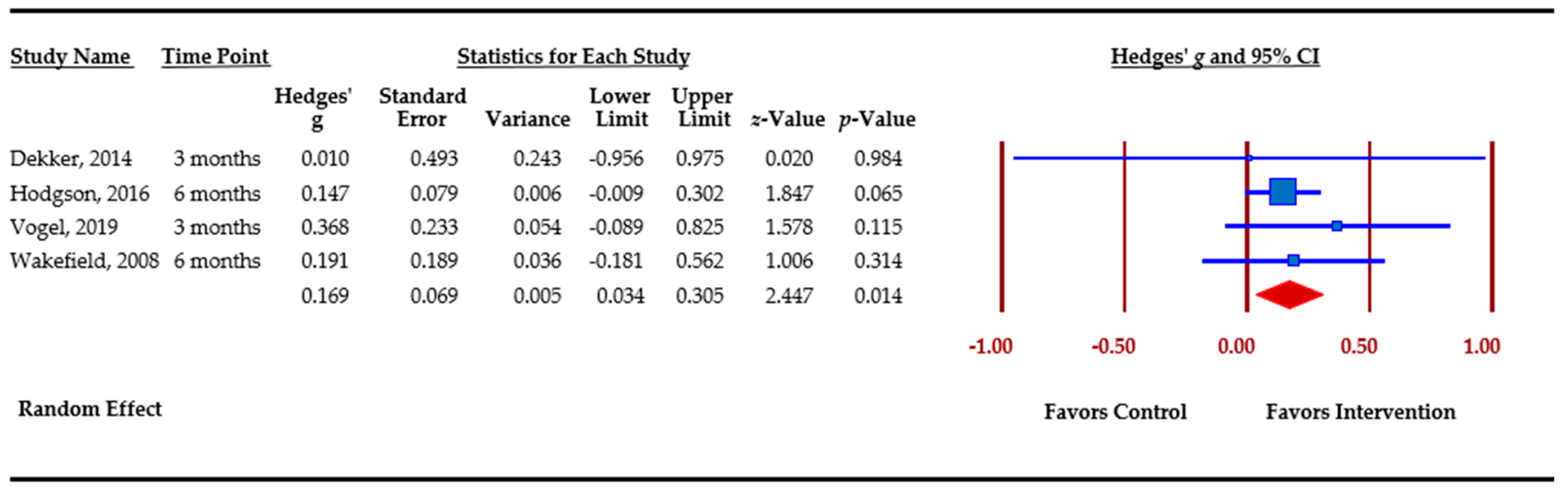

Figure 3. Cascade genetic testing.

Knowledge was conceptualized by primary studies as knowledge of heredity and cancer genetics, knowledge of risk factors and familial risk, and knowledge related to genetic testing. The $Q$ statistic indicates significant heterogeneity among the seven studies that evaluated changes in knowledge. The overall effect size was small but significant, $g=0.244(p<0.001)$, favoring the intervention arm. Effect sizes among primary studies varied between -0.273 and 0.708 (Figure 4 ).

\begin{tabular}{|c|c|c|c|c|c|c|c|c|}
\hline \multirow[t]{2}{*}{ Study Name } & \multirow[t]{2}{*}{ Time Point } & \multicolumn{7}{|c|}{ Statistics for Each Study } \\
\hline & & $\begin{array}{c}\text { Hedges' } \\
g\end{array}$ & $\begin{array}{l}\text { Standard } \\
\text { Error }\end{array}$ & Variance & $\begin{array}{l}\text { Lower } \\
\text { Limit }\end{array}$ & $\begin{array}{l}\text { Upper } \\
\text { Limit }\end{array}$ & $z$-Value & $p$-Value \\
\hline Dekker, 2014 & 3 months & 0.147 & 0.105 & 0.011 & -0.059 & 0.353 & 1.396 & 0.163 \\
\hline Eijzenga, 2018 & 4 months & -0.273 & 0.198 & 0.039 & -0.661 & 0.115 & -1.378 & 0.168 \\
\hline Katapodi, 2018 & 5 weeks & 0.047 & 0.635 & 0.403 & -1.198 & 1.292 & 0.074 & 0.941 \\
\hline Roshanai, 2009 & 2 weeks & 0.288 & 0.347 & 0.120 & -0.391 & 0.967 & 0.831 & 0.406 \\
\hline Tiller, 2006 & 2 weeks & 0.366 & 0.175 & 0.031 & 0.023 & 0.710 & 2.090 & 0.037 \\
\hline Vogel, 2019 & 3 months & 0.708 & 0.214 & 0.046 & 0.287 & 1.128 & 3.300 & 0.001 \\
\hline Wakefield, 2008 & 6 months & 0.516 & 0.185 & 0.034 & 0.154 & 0.878 & 2.794 & 0.005 \\
\hline & & 0.273 & 0.125 & 0.016 & 0.029 & 0.518 & 2.191 & 0.028 \\
\hline
\end{tabular}

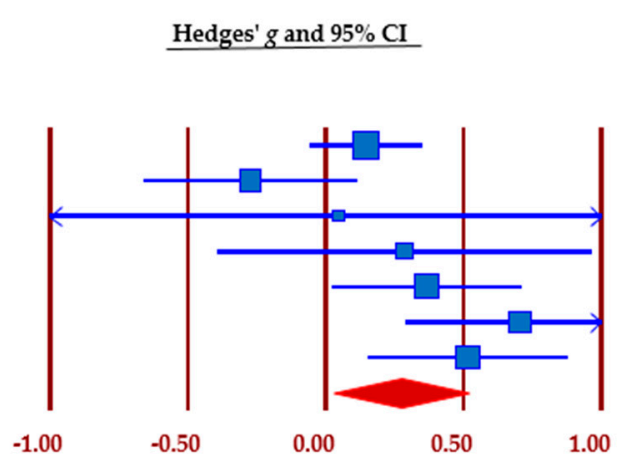

Favors Control

Favors Intervention

Figure 4. Knowledge.

Anxiety was assessed by primary studies with the Hospital Anxiety and Depression Scale (HADS) [55] and the Spielberger State Trait Anxiety Inventory (STAI) [56]. Egger's $t$-test indicates publication bias for the four studies that evaluated changes in anxiety. The overall effect size was small and not significant, $g=0.033(p=0.695)$. (Figure 5). 


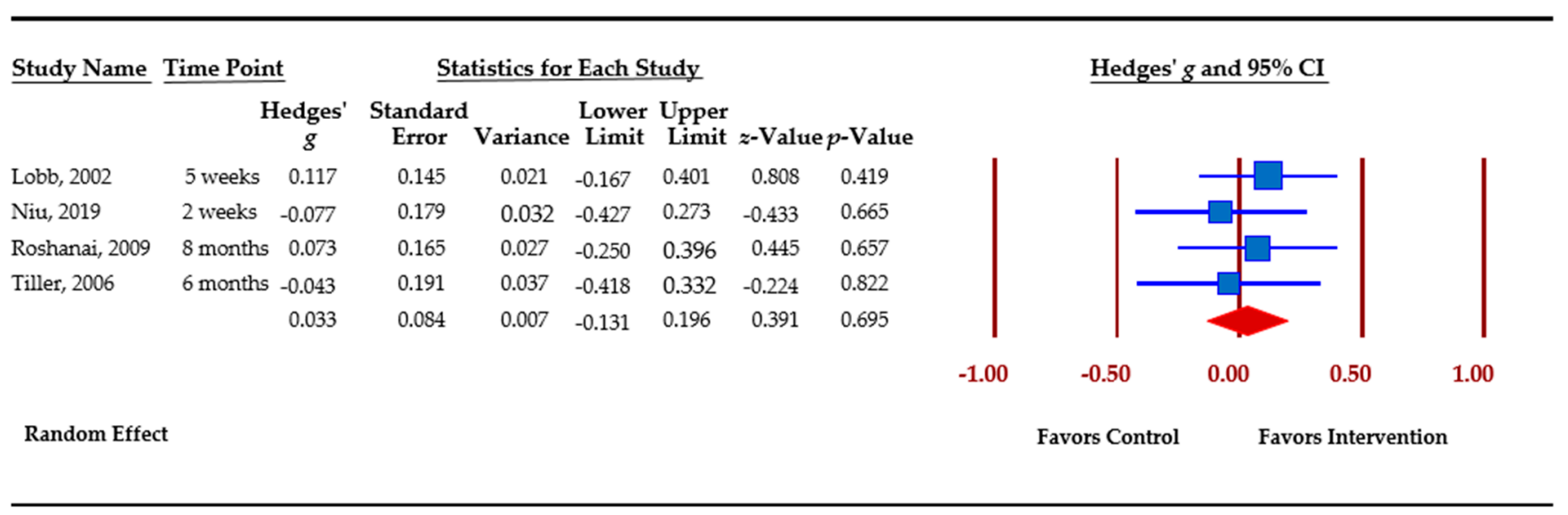

Figure 5. Anxiety.

Depression was assessed by primary studies using the Hospital Anxiety and Depression Scale (HADS) [55] and the Centers for Epidemiological Studies-Depression scale (CESD) [57]. Among the four studies that evaluated changes in depression, the overall effect size was small but significant, $g=0.183(p=0.017)$, favoring the intervention arm. Effect sizes among individual studies varied between 0.070 and 0.335 (Figure 6).

\begin{tabular}{|c|c|c|c|c|c|c|c|c|}
\hline \multirow[t]{2}{*}{ Study Name } & \multirow{2}{*}{$\underline{\text { Time Point }}$} & \multicolumn{7}{|c|}{$\underline{\text { Statistics for Each Study }}$} \\
\hline & & $\begin{array}{c}\text { Hedges' } \\
g\end{array}$ & $\begin{array}{c}\text { Standard } \\
\text { Error }\end{array}$ & Variance & $\begin{array}{l}\text { Lower } \\
\text { Limit }\end{array}$ & $\begin{array}{l}\text { Upper } \\
\text { Limit }\end{array}$ & $z$-Value & $p$-Value \\
\hline Lobb, 2002 & 5 weeks & 0.294 & 0.158 & 0.025 & -0.016 & 0.604 & 1.856 & 0.063 \\
\hline Montgomery, 2013 & 33 months & 0.070 & 0.127 & 0.016 & -0.179 & 0.319 & 0.554 & 0.580 \\
\hline Niu, 2019 & 2 weeks & 0.090 & 0.179 & 0.032 & -0.260 & 0.440 & 0.503 & 0.615 \\
\hline \multirow[t]{2}{*}{ Roshanai, 2009} & 2 weeks & 0.335 & 0.166 & 0.027 & 0.010 & 0.660 & 2.019 & 0.043 \\
\hline & & 0.183 & 0.077 & 0.006 & 0.033 & 0.334 & 2.387 & 0.017 \\
\hline
\end{tabular}

Random Effect

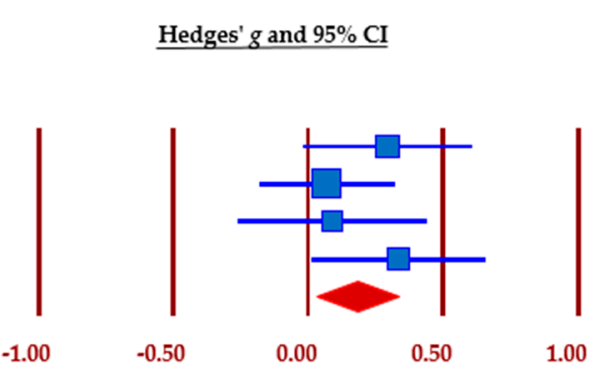

Favors Control

Favors Intervention

Figure 6. Depression.

Perceived risk for developing cancer was assessed in three studies. Changes in perceived risk were small and not significant, $g=0.007(p=0.95)$. (Figure 7).

Study Name Time Point

\begin{tabular}{|c|c|c|c|c|c|c|c|c|}
\hline & & $\underset{g}{\text { Hedges' }}$ & $\underset{\text { Error }}{\text { Standard }}$ & Variance & $\begin{array}{l}\text { Lower } \\
\text { Limit }\end{array}$ & $\begin{array}{c}\text { Upper } \\
\text { Limit }\end{array}$ & $z$-Value & $-\mathrm{V}$ \\
\hline Eijzenga, 2018 & 4 months & -0.070 & 0.138 & 0.019 & -0.340 & 0.200 & -0.506 & 0.613 \\
\hline Katapodi, 2018 & 5 weeks & 0.100 & 0.636 & 0.404 & -1.145 & 1.346 & 0.158 & 0.875 \\
\hline \multirow[t]{2}{*}{ Roshanai, 2009} & 2 weeks & 0.361 & 0.304 & 0.093 & -0.235 & 0.957 & 1.186 & 0.235 \\
\hline & & 0.007 & 0.123 & 0.015 & -0.234 & 0.248 & 0.058 & 0.954 \\
\hline
\end{tabular}

Random Effect
Hedges' $g$ and $95 \%$ CI

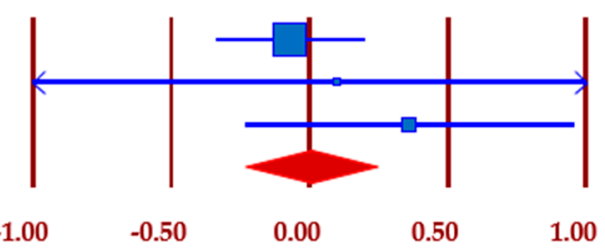

Favors Control Favors Intervention

Figure 7. Perceived risk. 


\section{Discussion}

The primary purpose of this paper was to identify interventions that were designed to facilitate family communication of cancer genetic testing results and/or cascade genetic testing among blood relatives, with a focus on HBOC and LS. To enhance the methodological rigor of our review, we focused exclusively on studies that tested intervention efficacy with an RCT design. Our systematic search identified 14 studies that met all inclusion criteria and were included in the narrative synthesis of this paper about intervention components, mode of delivery, and sample characteristics. Meta-analysis of outcomes was possible only for 13 studies, and not all of them had assessed family communication of test results and/or cascade genetic testing of relatives as a primary outcome. Our literature search identified serendipitously additional papers describing the development of relevant interventions [58-65]. However, none of them had been rigorously tested with an RCT, indicating that the scientific field is still in development. Our findings indicate that this is a growing field with significant heterogeneity of approaches, with few rigorously tested interventions that genetic professionals can emulate in cancer genetic practices.

The 14 identified interventions delivered to carriers of pathogenic variants and/or their blood relatives were comprehensive and addressed family communication, cascade testing of relatives, knowledge of cancer genetics, and psychosocial wellbeing as primary or secondary outcomes. We recorded three indicators of intervention quality. First, most studies included theory-driven intervention protocols, which decreases the likelihood of isolated or chance findings. However, there was considerable variability, with some studies mentioning the theory in passing or in generic terms, while others indicated specific theories and demonstrated how the theory was utilized in the selection of intervention content and choice of outcomes. Second, fewer studies instituted ways to examine intervention fidelity, i.e., the extent to which the protocol was delivered in a consistent manner. Investigators used protocol manuals, intervention logs, and/or tape-recorded sessions to assess or maintain intervention fidelity, indicating a growing understanding of the importance of adherence to standardized protocols. Third, there was considerable variability in intervention "dose" among protocols, both in the number of sessions (range 1 to 7 contacts) and duration of interventions (ranging from 20 to $140 \mathrm{~min}$, delivered over 7 days to 12 months). Detailed information about intervention dose was not consistently reported. Intervention dose could be further evaluated or standardized within studies; otherwise, it is difficult to determine if, or how much of, the intervention "dose" affects outcomes.

The majority of protocols included one-on-one and face-to-face or telephone extensive counseling with a trained healthcare provider, often identified as a genetic counselor or Master's-prepared nurse. Moreover, few interventions were delivered via a web-based or mobile app platform. Given the shortfall of trained genetic health professionals, technologybased approaches are needed to extend the reach to individuals weighing genetic testing decisions and facilitating cascade genetic testing. Increased access to genetic information could be facilitated with web-based or mobile health technologies. The availability of internet access, rising levels of electronic literacy, and the growing number of patient portals/web-based approaches hold promise for expanding the reach of tailored, costeffective genetic care $[27,66]$. Technology-enabled education and tele-genetics is equivalent to face-to-face consultations in presenting the benefits and drawbacks of genetic testing at half the cost of traditional consultations $[24,66]$.

Content related to the implications of genetic test results for blood relatives and communication was included in most interventions. However, the overall effect size for this outcome was small and not significant. There was significant heterogeneity among protocols, ranging from booklets that carriers could pass on to untested relatives, to family-based communication training. Some studies assessed communication as a secondary outcome. Taken together, these findings suggest that although building communication skills and/or providing support for dissemination of genetic testing results is an essential component, little is known about the best approach to enhance this outcome [67]. From the 14 protocols included in the narrative synthesis, many included extensive meetings with a healthcare 
provider, suggesting some individualization and tailoring of intervention content. However, most protocols targeted only carriers' communication skills and coping strategies, who are the transmitters of genetic information, and did not address communication and coping of relatives, who are the recipients. Communication of genetic test results is a twoway exchange between carriers and relatives and should be addressed as a family-based outcome, yet only two protocols included both a carrier and untested relatives. Enhancing communication of genetic testing results should be guided by family-based theoretical frameworks and tested with family-based designs [68-70].

When cascade genetic testing was the primary focus of interventions, the overall effect was small but significant. However, this finding should be interpreted with caution due to the small number of studies and the outcome based on self-reports. Invitation letters for genetic counseling, list of genetic resources, repeated contact with carriers over 12 months, and enhancing physician referrals were some of the techniques employed by the reviewed interventions. The current legal framework does not support healthcare professionals directly contacting blood relatives. However, removing this barrier does not guarantee successful cascading of blood relatives due to the resources needed to identify, contact, and counsel them. Additional measures, such as mailing of saliva kits [64] and family-based telephone or web-based counseling, hold promise to enhance cascade genetic testing and improve individual and population health outcomes.

Content related to cancer genetics, modes of inheritance, and risk factors was included in all interventions, resulting in a small but significant overall effect size and suggesting that this is an essential content area. This finding is consistent with an earlier review reporting that risk communication during genetic consultations increases genetic knowledge [71]. The significant heterogeneity observed for this outcome could be due to the different measures used to assess knowledge of cancer genetics, or due to the different syndromes and/or cancer types (e.g., colorectal or ovarian cancer) that were the focus of each intervention. Moreover, there is significant heterogeneity among counselees' preferences, with some preferring to receive detailed genetic information while others preferring "just the basics" [72,73], making streamlining lay genetic education difficult without a tailored approach.

Psychosocial outcomes, such as anxiety, depression, and perceived risk, as well as decisional conflict, regret, coping, and satisfaction were not assessed consistently among studies. Thus, we were unable to calculate pooled effect sizes for many of these outcomes. A significant number of interventions included decision aids, exercises for value clarification, and provided information on preventive and risk management options. These components likely enhance psychosocial adjustment to hereditary cancer risk and increase emotional wellbeing [71]. Although primary studies used validated instruments to assess these outcomes, meta-analysis findings regarding intervention efficacy, heterogeneity, and publication bias should be interpreted with caution due to the small number of primary studies and the heterogeneity of syndromes and/or cancer types. Risk communication in the clinical context resulted in general improvement for these outcomes [71].

Little is known about samples of racially, ethnically, and social diverse backgrounds. Only one study included a majority of Black participants, and only one study included a majority of male participants, indicating significant knowledge gaps regarding family communication and cascade genetic testing in males, especially in the context of HBOC. Future studies should also focus on LS, as it is the most common hereditary cancer condition known today, but remains largely undetected due to the different cancer types associated with LS and the lack of clear diagnostic criteria [74-77].

\section{Limitations}

We did not include studies published in languages other than English, unpublished studies, and abstracts from conference proceedings to ensure that findings were based on higher-quality, peer-reviewed studies. Excluding unpublished studies is likely to introduce an upward bias into the size of the effects found, which means that calculated effect sizes 
are likely to be larger [37]. To address this limitation, we assessed the heterogeneity of findings with the $Q$ statistic and publication bias with the Egger's $t$-test statistic. Publication bias appeared only in one outcome and may be related to the small number of studies. Our findings are comparable to a previous review assessing psychosocial outcomes of genetic counseling [62]. Finally, due to the small number of studies and the diverse outcomes, we were not able to conduct moderation analyses and examine the impact of similar types of interventions on outcomes (e.g., web-based vs. paper-based). The heterogeneity and attrition across studies also decrease our ability to discern the clinical utility of these interventions.

The time span of studies included in our meta-analysis covered a period of 17 years, during which there have been massive shifts in clinical practice and in public understanding of genetic testing. The introduction of panel testing has created new complexities in managing hereditary cancer risk associated with pathogenic variants of moderate penetrance, which may further contribute to existing barriers to family communication and cascade testing. GINA (Genetic Information Non-Discrimination Act), which was passed in the US in 2008 [78], may have lessened concerns about genetic discrimination, facilitating family communication and cascade genetic testing. However, this applies only to the seven studies conducted in the US, while the legal framework for protecting genetic information in other countries is not known. Discerning the influence of these two factors on family communication and cascade genetic testing is not possible under the scope of this study.

\section{Conclusions}

At the time of conducting this study, no similar reviews about family communication and/or cascade genetic testing for hereditary cancer syndromes have been published. Research has been mainly focused on helping healthcare professionals to facilitate family communication about genetic test results, and uptake of cascade testing has increased due to educational materials and technological resources and due to the active involvement of healthcare providers [79].

Although professional organizations call for the implementation of cascade testing for HBOC and LS, debate remains about the conflict between the need to protect the privacy of tested individuals and the rights of blood relatives to be notified about genetic information. Facilitating this process will contribute to the implementation of cascade genetic testing and significantly reduce the burden of cancer resulting from familial pathogenic variants. Technology- and theory-driven, rigorously-tested, psychoeducational interventions could play a significant role in this public health effort. Our study highlights the need for developing new interventions and new approaches in family communication and cascade testing for cancer susceptibility, laying the foundation for future work to address current knowledge gaps. Future studies could compare interventions assessing these outcomes regardless of the genetic condition, assuming similar "actionability" of genetic findings. Rigorous testing of promising interventions using an RCT design will propel the scientific field forward. In addition to individual- and family-level interventions, consideration should be given to health system and policy-level changes that might facilitate the communication of cancer genetic risk information and cascade testing.

Supplementary Materials: The following are available online at https:/ / www.mdpi.com/2072-669 4/13/4/925/s1, Table S1. Tabular representation of risk of bias in individual studies.

Author Contributions: Conceptualization, V.B., M.C.K.; methodology, V.B., M.C.K., M.L.U.-B., and C.A.-H.; software, V.B., M.C.K.; validation, V.B., M.L.U.-B., C.A.-H.; formal analysis, V.B., M.C.K.; investigation, V.B., M.L.U.-B., C.A.-H., M.C.K.; resources, M.C.K.; data curation, V.B.; writingoriginal draft preparation, V.B., M.C.K.; writing-review and editing, V.B., M.L.U.-B., C.A.-H., M.C.K.; visualization, V.B.; supervision M.C.K.; project administration V.B.; funding acquisition M.C.K. All authors have read and agreed to the published version of the manuscript.

Funding: This research was supported by the Swiss Cancer League (salary of VB and purchase of software) KLS-4294-08-2017. 
Acknowledgments: The authors would like to acknowledge Tarsha Jones, Florida Atlantic University, School of Nursing for her assistance in the original screening of identified articles and titles, and Hannah Ewald, University Medical Library Basel, for the peer review of search strategies.

Conflicts of Interest: The authors declare no conflict of interest.

\section{Appendix A}

Search Strategies

Embase.com

(20200615; 1,329 hits)

('hereditary breast and ovarian cancer syndrome' / de OR 'hereditary nonpolyposis colorectal cancer' / de OR 'BRCA1 protein'/de OR ‘BRCA2 protein' / de OR 'BRCA1 protein human' / de OR 'BRCA2 protein human' / de OR ‘BRCA gene' / de OR 'BRCA2 gene' / de OR 'BRCA protein' / de OR 'MutL protein homolog 1' / de OR 'mlh1 gene' / de OR 'mlh1 protein human' / de OR 'DNA mismatch repair protein MSH2' / de OR 'msh2 gene' / de OR 'msh2 protein human' / de OR 'protein MSH6' / de OR 'mismatch repair protein PMS2' / de OR 'pms2 gene' / de OR 'pms2 protein human' / de OR 'epithelial cell adhesion molecule' / de OR 'epcam gene' / de OR 'epcam protein human' / de OR ('HBOC syndrome' OR (('hereditary nonpolyposis' OR 'hereditary non polyposis') NEXT/3 (cancer OR neoplasm*)) OR HNPCC OR 'Lynch syndrome' OR 'Lynch II syndrome' OR 'Muir Torre syndrome' OR BRCA* OR FANCD* OR ('Fanconi anaemia' NEXT/3 D1) OR (MutL NEXT/2 'homolog 1') OR MLH1 OR hMLH1 OR ‘MutS homolog 2' OR MSH2 OR hMSH2 OR MSH6 OR 'postmeiotic segregation increased protein 2' OR PMS2 OR 'epithelial cell adhesion molecule' OR EPCAM):ab,ti OR ‘hereditary tumor syndrome' / de OR 'cancer risk' / de OR 'cancer susceptibility' / de OR 'oncogene' / de OR 'tumor suppressor gene' / de OR 'tumor gene' / de OR 'cancer genetics' / de OR (oncogene OR ((cancer* OR tumor* OR tumour*) NEAR/3 (syndrome OR risk OR predisposition OR susceptibility OR anticipation OR prognosis OR disorder OR gene*))):ab,ti OR (('genetic predisposition' / exp OR 'genetic risk' / de OR 'gene mutation' / de OR 'genetic disorder' / de OR 'single nucleotide polymorphism' / de OR 'family history' / de OR (hereditary OR inherit* OR inborn OR familial OR mutation* OR genetic ${ }^{*}$ OR ((family OR genomic*) NEAR/3 (syndrome OR risk OR predisposition OR disposition OR susceptibility OR anticipation OR prognosis OR disorder OR condition* OR history)) OR 'single nucleotide polymorphism*' OR SNP OR SNPs):ab,ti) AND ('neoplasm' / exp OR (cancer* OR neoplas* OR tumor* OR tumour* OR carcinoma* OR carcinogenesis OR malignan*):ab,ti)))

and

('counseling' / de OR 'genetic counseling' / de OR 'patient counseling' / de OR 'family counseling' / de OR 'consultation' / exp OR 'decision support system' / exp OR 'decision aid' / de OR 'interpersonal communication' / de OR 'persuasive communication' / de OR 'patient information' / de OR 'medical information' / de OR 'health education' / de OR 'patient education' / de OR 'education program' / de OR 'mass communication' / exp OR 'telephone interview' / de OR 'online system' / de OR 'questionnaire' / exp OR 'computer' / de OR 'compact disk' / exp OR 'mobile application' / exp OR 'website' / de OR 'multimedia' / de OR 'digital health' / de OR 'digital health technology' / de OR 'digital health intervention' / de OR 'telehealth' / exp OR 'mhealth' / de OR 'psychosocial care' / de OR 'social support' / de OR (((family OR genetic OR genomic OR patient* OR intervention OR risk*) NEAR/3 counsel $^{*}$ ) OR 'preventive genetics' OR consultation* OR teleconsultation* OR (decision NEAR/2 (support* OR aid* OR framework OR computer-assisted)) OR communicat* OR disclos* OR persuasion OR ((medical OR health OR patient* OR cancer OR risk* OR program ${ }^{*}$ ) NEAR/3 (information OR education)) OR internet OR ‘world wide web' OR web-based OR online OR 'social media' OR facebook OR twitter OR ((cell OR cellular OR mobile OR smart) NEXT phone*) OR cellphone* OR smartphone* OR mail OR (postal NEXT (delivery OR service)) OR letter* OR telehealth OR tele-health OR ehealth OR e-health OR 'digital health' OR mhealth OR telemedicine OR tele-medicine OR telephone* OR dataphone* OR videoconferenc* OR ‘video conferenc*' OR webcast OR computer* OR questionnaire* OR 
survey* OR 'compact disk' OR 'CD-I' OR ‘CD-ROM' OR DVD OR ((mobile OR portable OR educational) NEXT/2 (app OR apps OR application*)) OR website* OR 'web site* OR multimedia OR ((psychosocial OR social) NEXT (care OR support OR therapy OR intervention*)) OR telegenetic OR 'educational material ${ }^{* \prime}$ OR 'tailored message ${ }^{* \prime}$ OR 'message tailoring'):ab,ti)

and

('genetic service'/exp OR 'genetic analysis'/de OR 'mutational analysis' /exp OR 'DNA sequencing'/de OR 'genetic discrimination' / de OR 'genetic diagnosis' / de OR 'family therapy' / de OR 'family counseling' / de OR 'family study'/de OR 'informed decision making'/de OR 'informed choice' / de OR (((family OR genetic* OR genomic* OR cascade OR mutation*) NEAR/3 (counsel* OR care OR testing OR screening OR analys* OR study OR studies OR discrimination OR diagnos*)) OR 'DNA sequencing' OR 'preventive genetics' OR ((family OR relative*) NEAR/3 (communicat* OR intervention* OR inform*)) OR (informed NEXT (decision* OR choice*))):ab,ti)

and

('crossover procedure':de OR 'double-blind procedure':de OR 'randomized controlled trial':de OR 'single-blind procedure':de OR (random* OR factorial ${ }^{*}$ OR crossover* OR cross NEXT / 1 over* OR placebo* OR doubl ${ }^{*}$ NEAR/1 blind* OR singl* NEAR/1 blind* OR

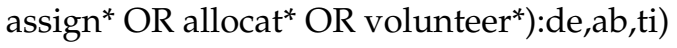

Medline (Ovid)

(20200615; 1,566 hits)

("hereditary breast and ovarian cancer syndrome" /OR Colorectal Neoplasms, Hereditary Nonpolyposis/OR BRCA1 protein/OR BRCA1 protein, human.nm. OR BRCA2 protein/OR BRCA2 protein, human.nm. OR Genes, BRCA1/OR Genes, BRCA2/OR exp MutL proteins/OR MLH1 protein, human.nm. OR G-T mismatch-binding protein.nm. OR MSH2 protein, human.nm. OR exp MutS proteins/OR PMS2 protein, human.nm. OR epithelial cell adhesion molecule/OR EPCAM protein, human.nm. OR (HBOC syndrome OR ((hereditary nonpolyposis OR hereditary non polyposis) ADJ3 (cancer OR neoplasm*)) OR HNPCC OR Lynch syndrome OR Lynch II syndrome OR Muir Torre syndrome OR BRCA* OR FANCD* OR (Fanconi anaemia ADJ3 D1) OR (MutL ADJ2 homolog 1) OR MLH1 OR hMLH1 OR MutS homolog 2 OR MSH2 OR hMSH2 OR MSH6 OR postmeiotic segregation increased protein 2 OR PMS2 OR epithelial cell adhesion molecule OR EPCAM).ab,ti. OR Neoplastic Syndromes, Hereditary/OR oncogenes/OR Genes, Tumor Suppressor/OR Genes, Neoplasm/OR (oncogene OR ((cancer* OR tumor* OR tumour*) ADJ3 (syndrome OR risk OR predisposition OR susceptibility OR anticipation OR prognosis OR disorder OR gene $\left.\left.e^{*}\right)\right)$.ab,ti. OR ((exp Genetic Predisposition to Disease/OR mutation/OR Genetic Diseases, Inborn/OR Polymorphism, Single Nucleotide/OR (hereditary OR inherit* OR inborn OR familial OR mutation* OR genetic* OR ((family OR genomic*) ADJ3 (syndrome OR risk OR predisposition OR disposition OR susceptibility OR anticipation OR prognosis OR disorder OR condition* OR history)) OR single nucleotide polymorphism* OR SNP OR SNPs).ab,ti.) AND (exp neoplasms/OR (cancer* OR neoplas* OR tumor* OR tumour* OR carcinoma* OR carcinogenesis OR malignan*).ab,ti.)))

and

(counseling/OR genetic counseling/OR Referral and Consultation/OR exp Remote Consultation/OR decision support systems, clinical/OR Decision Support Techniques/OR communication/OR persuasive communication/OR health education/OR patient education as topic/OR exp telecommunications/OR Interviews as Topic/OR online systems/OR "Surveys and Questionnaires" /OR computers/OR exp compact disks/OR mobile applications/OR multimedia/OR telemedicine/OR exp social support/OR ((family OR genetic OR genomic OR patient ${ }^{*}$ OR intervention OR risk ${ }^{*}$ ADJ3 counsel ${ }^{*}$ ) OR preventive genetics OR consultation* OR teleconsultation* OR (decision ADJ2 (support* OR aid* OR framework OR computer-assisted)) OR communicat ${ }^{*}$ OR disclos* OR persuasion OR ((medical OR health OR patient* OR cancer OR risk* OR program*) ADJ3 (information OR education)) OR internet OR world wide web OR web-based OR online OR social media OR facebook 
OR twitter OR ((cell OR cellular OR mobile OR smart) ADJ phone*) OR cellphone* OR smartphone* OR mail OR (postal ADJ (delivery OR service)) OR letter* OR telehealth OR tele-health OR ehealth OR e-health OR digital health OR mhealth OR telemedicine OR tele-medicine OR telephone ${ }^{*}$ OR dataphone* OR videoconferenc* ${ }^{*}$ OR video conferenc ${ }^{*}$ OR webcast OR computer* OR questionnaire* OR survey* OR compact disk OR CD-I OR CD-ROM OR DVD OR ((mobile OR portable OR educational) ADJ2 (app OR apps OR application*)) OR website* OR web site* OR multimedia OR ((psychosocial OR social) ADJ (care OR support OR therapy OR intervention*)) OR telegenetic OR educational material* OR tailored message* OR message tailoring).ab,ti.)

and

(exp genetic services/OR DNA mutational analysis/OR Sequence Analysis, DNA/OR family therapy/OR (((family OR genetic* OR genomic* OR cascade OR mutation*) ADJ3 (counsel* OR care OR testing OR screening OR analys* OR study OR studies OR discrimination OR diagnos*)) OR DNA sequencing OR preventive genetics OR ((family OR relative $^{*}$ ) ADJ3 (communicat* OR intervention* OR inform*)) OR (informed ADJ (decision* OR choice $\left.\left.{ }^{*}\right)\right)$.ab,ti.)

and

(randomized controlled trial.pt. OR controlled clinical trial.pt. OR randomized.ti,ab. OR placebo.ti,ab. OR drug therapy.fs. OR randomly.ti,ab. OR trial.ti,ab. OR groups.ti,ab. NOT (exp animals/NOT exp humans/))

\section{CENTRAL}

(20200615; 652 hits)

(('HBOC syndrome' OR (('hereditary nonpolyposis' OR 'hereditary non polyposis') NEXT/3 (cancer OR neoplasm*)) OR HNPCC OR 'Lynch syndrome' OR 'Lynch II syndrome' OR 'Muir Torre syndrome' OR BRCA* OR FANCD' OR ('Fanconi anaemia' NEXT/3 D1) OR (MutL NEXT / 2 'homolog 1') OR MLH1 OR hMLH1 OR 'MutS homolog 2' OR MSH2 OR hMSH2 OR MSH6 OR 'postmeiotic segregation increased protein 2' OR PMS2 OR 'epithelial cell adhesion molecule' OR EPCAM):ab,ti OR (oncogene OR ((cancer* OR tumor* OR tumour*) NEAR/3 (syndrome OR risk OR predisposition OR susceptibility OR anticipation OR prognosis OR disorder OR gene $\left.\left.e^{*}\right)\right)$ :ab,ti OR (((hereditary OR inherit* OR inborn OR familial OR mutation* OR genetic* OR ((family OR genomic*) NEAR/3 (syndrome OR risk OR predisposition OR disposition OR susceptibility OR anticipation OR prognosis OR disorder OR condition* OR history)) OR 'single nucleotide polymorphism*' OR SNP OR SNPs):ab,ti) AND ((cancer* OR neoplas* OR tumor* OR tumour* OR carcinoma* OR carcinogenesis OR malignan*):ab,ti))) AND ((((family OR genetic OR genomic OR patient* OR intervention OR risk $\left.{ }^{*}\right) \mathrm{NEAR} / 3$ counsel $^{*}$ ) OR 'preventive genetics' OR consultation* OR teleconsultation* OR (decision NEAR/2 (support* OR aid* OR framework OR computerassisted)) OR communicat* OR disclos* OR persuasion OR ((medical OR health OR patient* OR cancer OR risk* OR program*) NEAR/3 (information OR education)) OR internet OR 'world wide web' OR web-based OR online OR 'social media' OR facebook OR twitter OR ((cell OR cellular OR mobile OR smart) NEXT phone*) OR cellphone* OR smartphone* OR mail OR (postal NEXT (delivery OR service)) OR letter* OR telehealth OR tele-health OR ehealth OR e-health OR 'digital health' OR mhealth OR telemedicine OR tele-medicine OR telephone* OR dataphone* OR videoconferenc* OR 'video conferenc*' OR webcast OR computer* OR questionnaire* OR survey* OR 'compact disk' OR 'CD-I' OR 'CD-ROM' OR DVD OR ((mobile OR portable OR educational) NEXT/2 (app OR apps OR application*)) OR website* OR 'web site*' OR multimedia OR ((psychosocial OR social) NEXT (care OR support OR therapy OR intervention*)) OR telegenetic OR 'educational material ${ }^{* \prime}$ OR 'tailored message ${ }^{* \prime}$ OR 'message tailoring'):ab,ti) AND ((((family OR genetic* OR genomic ${ }^{*}$ OR cascade OR mutation*) NEAR/3 (counsel* OR care OR testing OR screening OR analys* OR study OR studies OR discrimination OR diagnos*)) OR ‘DNA sequencing' OR 'preventive genetics' OR ((family OR relative $\left.{ }^{*}\right)$ NEAR/3 (communicat* OR intervention* OR inform*)) OR (informed NEXT (decision* OR choice*))):ab,ti)

PsycInfo 
(20200615; 123 hits)

((HBOC syndrome OR ((hereditary nonpolyposis OR hereditary non polyposis) ADJ3 (cancer OR neoplasm*)) OR HNPCC OR Lynch syndrome OR Lynch II syndrome OR Muir Torre syndrome OR BRCA* OR FANCD* OR (Fanconi anaemia ADJ3 D1) OR (MutL ADJ2 homolog 1) OR MLH1 OR hMLH1 OR MutS homolog 2 OR MSH2 OR hMSH2 OR MSH6 OR postmeiotic segregation increased protein 2 OR PMS2 OR epithelial cell adhesion molecule OR EPCAM).ab,ti. OR (oncogene OR ((cancer* OR tumor* OR tumour*) ADJ3 (syndrome OR risk OR predisposition OR susceptibility OR anticipation OR prognosis OR disorder OR gene $\left.\left.\left.{ }^{*}\right)\right)\right)$.ab,ti. OR (((Genetics/AND Predisposition/) OR mutations/OR Genetic Disorders/OR At Risk Populations/OR (hereditary OR inherit* OR inborn OR familial OR mutation* OR genetic* OR ((family OR genomic*) ADJ3 (syndrome OR risk OR predisposition OR disposition OR susceptibility OR anticipation OR prognosis OR disorder OR condition* OR history)) OR single nucleotide polymorphism* OR SNP OR SNPs).ab,ti.) AND (exp neoplasms/OR (cancer* OR neoplas* OR tumor* OR tumour* OR carcinoma* OR carcinogenesis OR malignan*).ab,ti.)))

and

(counseling/OR genetic counseling/OR Professional Consultation/OR decision support systems/OR exp interpersonal communication/OR persuasive communication/OR health education/OR Interviews/OR Questionnaires/OR internet/OR exp computers/OR mobile applications/OR multimedia/OR exp telemedicine/OR social support/OR (((family OR genetic OR genomic OR patient* OR intervention OR risk*) ADJ3 counsel ${ }^{*}$ ) OR preventive genetics OR consultation* OR teleconsultation* OR (decision ADJ2 (support* OR aid* OR framework OR computer-assisted)) OR communicat ${ }^{*}$ OR disclos* OR persuasion OR ((medical OR health OR patient* OR cancer OR risk* OR program*) ADJ3 (information OR education)) OR internet OR world wide web OR web-based OR online OR social media OR facebook OR twitter OR ((cell OR cellular OR mobile OR smart) ADJ phone*) OR cellphone* OR smartphone* OR mail OR (postal ADJ (delivery OR service)) OR letter* OR telehealth OR tele-health OR ehealth OR e-health OR digital health OR mhealth OR telemedicine OR tele-medicine OR telephone* OR dataphone* OR videoconferenc ${ }^{*}$ OR video conferenc* OR webcast OR computer* OR questionnaire* OR survey* OR compact disk OR CD-I OR CD-ROM OR DVD OR ((mobile OR portable OR educational) ADJ2 (app OR apps OR application*)) OR website* OR web site* OR multimedia OR ((psychosocial OR social) ADJ (care OR support OR therapy OR intervention*)) OR telegenetic OR educational material* OR tailored message* OR message tailoring).ab,ti.)

and

(genetic counseling/OR genetic testing/OR exp family therapy/OR (((family OR genetic* OR genomic $^{*}$ OR cascade OR mutation*) ADJ3 (counsel* OR care OR testing OR screening OR analys* OR study OR studies OR discrimination OR diagnos*)) OR DNA sequencing OR preventive genetics OR ((family OR relative*) ADJ3 (communicat* OR intervention* OR inform*)) OR (informed ADJ (decision* OR choice*))).ab,ti.)

and

((Randomized Controlled Trial OR Controlled Clinical Trial OR Pragmatic Clinical Trial OR Equivalence Trial OR Clinical Trial, Phase III).pt. OR Randomized Controlled Trial/OR exp Randomized Controlled Trials as Topic/OR "Randomized Controlled Trial (topic)" /OR Controlled Clinical Trial/OR exp Controlled Clinical Trials as Topic/OR "Controlled Clinical Trial (topic)"/OR Randomization/OR Random Allocation/OR Double-Blind Method/OR Double Blind Procedure/OR Double-Blind Studies/OR Single-Blind Method/OR Single Blind Procedure/OR Single-Blind Studies/OR Placebos/OR Placebo/OR Control Groups/OR Control Group/OR (random* OR sham OR placebo*).ti,ab,hw. OR ((singl* OR doubl $\left.{ }^{*}\right)$ ADJ (blind* OR dumm* OR mask*)).ti,ab,hw. OR ((tripl ${ }^{*}$ OR trebl*) ADJ (blind* OR dumm* OR mask*)).ti,ab,hw. OR (control* ADJ3 (study OR studies OR trial* OR group*)).ti,ab. OR (Nonrandom* OR non random* OR non-random* OR quasi-random* OR quasirandom*).ti,ab,hw. OR allocated.ti,ab,hw. OR ((open label OR open-label) ADJ5 (study OR studies OR trial*)).ti,ab,hw. OR ((equivalence 
OR superiORity OR non-inferiORity OR noninferiORity) ADJ3 (study OR studies OR trial $\left.^{*}\right)$ ).ti,ab,hw. OR (pragmatic study OR pragmatic studies).ti,ab,hw. OR ((pragmatic OR practical) ADJ3 trial*).ti,ab,hw. OR ((quasiexperimental OR quasi-experimental) ADJ3 (study OR studies OR trial*)).ti,ab,hw. OR (phase ADJ3 (III OR “3") ADJ3 (study OR studies OR trial $\left.^{*}\right)$ ).ti,hw.)

\section{References}

1. Ferlay, J.; Soerjomataram, I.; Dikshit, R.; Eser, S.; Mathers, C.; Rebelo, M.; Parkin, D.M.; Forman, D.; Bray, F. Cancer incidence and mortality worldwide: Sources, methods and major patterns in Globocan 2012. Int. J. Cancer 2015, 136, E359-E386. [CrossRef]

2. Allemani, C.; Weir, H.K.; Carreira, H.; Harewood, R.; Spika, D.; Wang, X.S.; Bannon, F.; Ahn, J.V.; Johnson, C.J.; Bonaventure, A.; et al. Global surveillance of cancer survival 1995-2009: Analysis of individual data for 25,676,887 patients from 279 populationbased registries in 67 countries (Concord-2). Lancet 2015, 385, 977-1010. [CrossRef]

3. Hu, C.; Hart, S.N.; Gnanaolivu, R.; Huang, H.; Lee, K.Y.; Na, J.; Gao, C.; Lilyquist, J.; Yadav, S.; Boddicker, N.J.; et al. A population-based study of genes previously implicated in breast cancer. N. Engl. J. Med. 2021, 384, 440-451. [CrossRef] [PubMed]

4. Chubb, D.; Broderick, P.; Frampton, M.; Kinnersley, B.; Sherborne, A.; Penegar, S.; Lloyd, A.; Ma, Y.P.; Dobbins, S.E.; Houlston, R.S. Genetic diagnosis of high-penetrance susceptibility for colorectal cancer (CRC) is achievable for a high proportion of familial CRC by exome sequencing. J. Clin. Oncol. 2015, 33, 426-432. [CrossRef] [PubMed]

5. Walsh, T.; Casadei, S.; Lee, M.K.; Pennil, C.C.; Nord, A.S.; Thornton, A.M.; Roeb, W.; Agnew, K.J.; Stray, S.M.; Wickramanayake, A.; et al. Mutations in 12 genes for inherited ovarian, fallopian tube, and peritoneal carcinoma identified by massively parallel sequencing. Proc. Natl. Acad. Sci. USA 2011, 108, 18032-18037. [CrossRef]

6. Couch, F.J.; Nathanson, K.L.; Offit, K. Two decades after BRCA: Setting paradigms in personalized cancer care and prevention. Science 2014, 343, 1466-1470. [CrossRef] [PubMed]

7. Mavaddat, N.; Peock, S.; Frost, D.; Ellis, S.; Platte, R.; Fineberg, E.; Evans, D.G.; Izatt, L.; Eeles, R.A.; Adlard, J.; et al. Cancer risks for BRCA1 and BRCA2 mutation carriers: Results from prospective analysis of embrace. J. Natl. Cancer Inst. 2013, 105, 812-822. [CrossRef]

8. Giardiello, F.M.; Allen, J.I.; Axilbund, J.E.; Boland, C.R.; Burke, C.A.; Burt, R.W.; Church, J.M.; Dominitz, J.A.; Johnson, D.A.; Kaltenbach, T.; et al. Guidelines on genetic evaluation and management of lynch syndrome: A consensus statement by the us multi-society task force on colorectal cancer. Am. J. Gastroenterol. 2014, 109, 1159-1179. [CrossRef]

9. Bellcross, C.A.; Bedrosian, S.R.; Daniels, E.; Duquette, D.; Hampel, H.; Jasperson, K.; Joseph, D.A.; Kaye, C.; Lubin, I.; Meyer, L.J.; et al. Implementing screening for Lynch syndrome among patients with newly diagnosed colorectal cancer: Summary of a public health/clinical collaborative meeting. Genet. Med. 2012, 14, 152-162. [CrossRef]

10. Syngal, S.; Brand, R.E.; Church, J.M.; Giardiello, F.M.; Hampel, H.L.; Burt, R.W. Acg clinical guideline: Genetic testing and management of hereditary gastrointestinal cancer syndromes. Am. J. Gastroenterol. 2015, 110, 223-262. [CrossRef] [PubMed]

11. Bibbins-Domingo, K.; Grossman, D.C.; Curry, S.J.; Davidson, K.W.; Epling, J.W., Jr.; Garcia, F.A.; Gillman, M.W.; Harper, D.M.; Kemper, A.R.; Krist, A.H.; et al. Screening for colorectal cancer: US preventive services task force recommendation statement. JAMA 2016, 315, 2564-2575. [PubMed]

12. Greenberg, S.; Buys, S.S.; Edwards, S.L.; Espinel, W.; Fraser, A.; Gammon, A.; Hafen, B.; Herget, K.A.; Kohlmann, W.; Roundy, C.; et al. Population prevalence of individuals meeting criteria for hereditary breast and ovarian cancer testing. Cancer Med. 2019, 8, 6789-6798. [CrossRef]

13. Grosse, S.D.; Rogowski, W.H.; Ross, L.F.; Cornel, M.C.; Dondorp, W.J.; Khoury, M.J. Population screening for genetic disorders in the 21st century: Evidence, economics, and ethics. Public Health Genom. 2010, 13, 106-115. [CrossRef]

14. Clain, E.; Trosman, J.R.; Douglas, M.P.; Weldon, C.B.; Phillips, K.A. Availability and payer coverage of BRCA1/2 tests and gene panels. Nat. Biotechnol. 2015, 33, 900-902. [CrossRef]

15. Hampel, H. Genetic counseling and cascade genetic testing in Lynch syndrome. Fam. Cancer 2016, 15, 423-427. [CrossRef] [PubMed]

16. Khoury, M.J.; Evans, J.P. A public health perspective on a national precision medicine cohort: Balancing long-term knowledge generation with early health benefit. JAMA 2015, 313, 2117-2118. [CrossRef] [PubMed]

17. Fraser, L.; Bramald, S.; Chapman, C.; Chu, C.; Cornelius, V.; Douglas, F.; Lucassen, A.; Nehammer, A.; Sutton, S.; Trivella, M.; et al. What motivates interest in attending a familial cancer genetics clinic? Fam. Cancer 2003, 2, 159-168. [CrossRef]

18. Van den Heuvel, M.L.; Stemkens, D.; van Zelst-Stams, W.A.G.; Willeboordse, F.; Christiaans, I. How to inform at-risk relatives? Attitudes of 1379 Dutch patients, relatives, and members of the general population. J. Genet. Couns. 2020, 29, 786-799. [CrossRef]

19. Daly, M.B.; Montgomery, S.; Bingler, R.; Ruth, K. Communicating genetic test results within the family: Is it lost in translation? A survey of relatives in the randomized six-step study. Fam. Cancer 2016, 15, 697-706. [CrossRef]

20. Taber, J.M.; Chang, C.Q.; Lam, T.K.; Gillanders, E.M.; Hamilton, J.G.; Schully, S.D. Prevalence and correlates of receiving and sharing high-penetrance cancer genetic test results: Findings from the health information national trends survey. Public Health Genom. 2015, 18, 67-77. [CrossRef]

21. Fadda, M.; Chappuis, P.O.; Katapodi, M.C.; Pagani, O.; Monnerat, C.; Membrez, V.; Unger, S.; Caiata Zufferey, M. Physicians communicating with women at genetic risk of breast and ovarian cancer: Are we in the middle of the ford between contradictory messages and unshared decision making? PLoS ONE 2020, 15, e0240054. [CrossRef] [PubMed] 
22. Bowen, D.J.; Hyams, T.; Laurino, M.; Woolley, T.; Cohen, S.; Leppig, K.A.; Jarvik, G. Development of FamilyTalk: An intervention to support communication and educate families about colorectal cancer risk. J. Cancer Educ. 2019, 35, 470-478. [CrossRef] [PubMed]

23. Birch, P.H. Interactive e-counselling for genetics pre-test decisions: Where are we now? Clin. Genet. 2015, 87, 209-217. [CrossRef] [PubMed]

24. Syrowatka, A.; Krömker, D.; Meguerditchian, A.N.; Tamblyn, R. Features of computer-based decision aids: Systematic review, thematic synthesis, and meta-analyses. J. Med. Internet Res. 2016, 18, e20. [CrossRef] [PubMed]

25. Biesecker, B.B.; Lewis, K.L.; Umstead, K.L.; Johnston, J.J.; Turbitt, E.; Fishler, K.P.; Patton, J.H.; Miller, I.M.; Heidlebaugh, A.R.; Biesecker, L.G. Web platform vs. in-person genetic counselor for return of carrier results from exome sequencing: A randomized clinical trial. JAMA Intern. Med. 2018, 178, 338-346. [CrossRef]

26. Otten, E.; Birnie, E.; Ranchor, A.V.; van Langen, I.M. Telegenetics use in presymptomatic genetic counselling: Patient evaluations on satisfaction and quality of care. Eur. J. Hum. Genet. 2016, 24, 513. [CrossRef]

27. Buchanan, A.H.; Datta, S.K.; Skinner, C.S.; Hollowell, G.P.; Beresford, H.F.; Freeland, T.; Rogers, B.; Boling, J.; Marcom, P.K.; Adams, M.B. Randomized trial of telegenetics vs. in-person cancer genetic counseling: Cost, patient satisfaction and attendance. J. Genet. Couns. 2015, 24, 961-970. [CrossRef]

28. Gaieski, J.B.; Patrick-Miller, L.; Egleston, B.L.; Maxwell, K.N.; Walser, S.; DiGiovanni, L.; Brower, J.; Fetzer, D.; Ganzak, A.; McKenna, D.; et al. Research participants' experiences with return of genetic research results and preferences for web-based alternatives. Mol. Genet. Genom. Med. 2019, 7, e898. [CrossRef]

29. Moher, D.; Liberati, A.; Tetzlaff, J.; Altman, D.G. Preferred reporting items for systematic reviews and meta-analyses: The PRISMA statement. PLoS Med 2009, 6, e1000097. [CrossRef]

30. Lefebvre, C.; Manheimer, E.; Glanville, J. Chapter 6: Searching for studies. Cochrane Handbook for Systematic Reviews of Interventions. Version 5.1.0 (updated 11); Higgins, J., Green, S., Eds.; The Cochrane Collaboration, 2011. Available online: www.cochranehandbook.org.

31. CADTH database search filters (Internet). Ottawa: CADTH; 2016. Available online: https://www.cadth.ca/resources/findingevidence (accessed on 31 January 2021).

32. Endnote, Version EndNote X9; Clarivate: Philadelphia, PA, USA, 2018.

33. Bramer, W.M.; Giustini, D.; de Jonge, G.B.; Holland, L.; Bekhuis, T. De-duplication of database search results for systematic reviews in EndNote [published correction appears in J Med Libr Assoc. 2017 Jan;105(1):111]. J. Med. Libr. Assoc. 2016, 104, 240-243. [CrossRef] [PubMed]

34. Covidence Systematic Review Software, Veritas Health Innovation, Melbourne, Australia. Available online: www.covidence.org (accessed on 25 August 2020).

35. Higgins, J.P.T.; Altman, D.G.; Sterne, J.A.C. Assessing risk of bias in included studies. In Cochrane Handbook for Systematic Reviews of Interventions, Version 5.2.0; Cochrane: London, UK, 2017.

36. Borenstein, M.; Hedges, L.V.; Higgins, J.P.T.; Rothstein, H.R. Introduction to Meta-Analysis; Wiley: Hoboken, NJ, USA, 2009; pp. $1-421$.

37. Lipsey, M.W.; Wilson, D.B. Practical Meta-Analysis; SAGE Publications, Inc.: Thousand Oaks, CA, USA, 2001.

38. Cohen, J. Statistical Power Analysis for the Behavioral Sciences; Academic Press: Cambridge, MA, USA, 2013.

39. Rosenthal, R. Meta-Analytic Procedures for Social Research; SAGE Publications, Inc.: Thousand Oaks, CA, USA, 1991.

40. Comprehensive Meta-Analysis, Version 3; Biostat Inc.: Englewood, NJ, USA, 2020.

41. McInerney, L.A.; Biesecker, B.B.; Hadley, D.W.; Kase, R.G.; Giambarresi, T.R.; Johnson, E.; Lerman, C.; Struewing, J.P. BRCA1/2 testing in hereditary breast and ovarian cancer families: Effectiveness of problem-solving training as a counseling intervention. Am. J. Med. Genet. A 2004, 130a, 221-227. [CrossRef] [PubMed]

42. Katapodi, M.C.; Jung, M.; Schafenacker, A.M.; Milliron, K.J.; Mendelsohn-Victor, K.E.; Merajver, S.D.; Northouse, L.L. Development of a family-based intervention for BRCA carriers and their at-risk relatives: Focus groups, usability, and feasibility testing. JMIR Cancer 2018, 4, e7. [CrossRef]

43. Lobb, E.; Butow, P.; Meiser, B.; Barratt, A.; Kirk, J.; Gattas, M.; Haan, E.; Tucker, K. The use of audiotapes in consultations with women from high risk breast cancer families: A randomised trial. J. Med. Genet. 2002, 39, 697-703. [CrossRef]

44. Montgomery, S.V.; Barsevick, A.M.; Egleston, B.L.; Bingler, R.; Ruth, K.; Miller, S.M.; Malick, J.; Cescon, T.P.; Daly, M.B. preparing individuals to communicate genetic test results to their relatives: Report of a randomized control trial. Fam. Cancer 2013, 12, 537-546. [CrossRef]

45. Wakefield, C.E.; Meiser, B.; Homewood, J.; Peate, M.; Taylor, A.; Lobb, E.; Kirk, J.; Young, M.A.; Williams, R.; Dudding, T.; et al. A randomized controlled trial of a decision aid for women considering genetic testing for breast and ovarian cancer risk. Breast Cancer Res. Treat. 2008, 107, 289-301. [CrossRef]

46. Bodurtha, J.N.; McClish, D.; Gyure, M.; Corona, R.; Krist, A.H.; Rodríguez, V.M.; Maibauer, A.M.; Borzelleca, J., Jr.; Bowen, D.J.; Quillin, J.M. The kinfact intervention-A randomized controlled trial to increase family communication about cancer history. J. Womens Health 2014, 23, 806-816. [CrossRef] [PubMed]

47. Eijzenga, W.; de Geus, E.; Aalfs, C.M.; Menko, F.H.; Sijmons, R.H.; de Haes, H.C.J.M.; Smets, E.M.A. How to support cancer genetics counselees in informing at-risk relatives? Lessons from a randomized controlled trial. Patient Educ. Couns. 2018, 101, 1611-1619. [CrossRef] [PubMed] 
48. Hodgson, J.; Metcalfe, S.; Gaff, C.; Donath, S.; Delatycki, M.B.; Winship, I.; Skene, L.; Aitken, M.A.; Halliday, J. Outcomes of a randomised controlled trial of a complex genetic counselling intervention to improve family communication. Eur. J. Human Genet. 2016, 24, 356-360. [CrossRef]

49. Roshanai, A.H.; Rosenquist, R.; Lampic, C.; Nordin, K. Does enhanced information at cancer genetic counseling improve counselees' knowledge, risk perception, satisfaction and negotiation of information to at-risk relatives?-A randomized study. Acta Oncologica 2009, 48, 999-1009. [CrossRef]

50. Dekker, N.; Hermens, R.P.; de Wilt, J.H.; van Zelst-Stams, W.A.; Hoogerbrugge, N.; Nagengast, F.; Elwyn, G.; Ligtenberg, M.; van Krieken, J.; Adang, E.; et al. Improving recognition and referral of patients with an increased familial risk of colorectal cancer: Results from a randomized controlled trial. Colorectal Dis. 2015, 17, 499-510. [CrossRef]

51. Loader, S.; Shields, C.; Levenkron, J.C.; Fishel, R.; Rowley, P.T. Patient vs. physician as the target of educational outreach about screening for an inherited susceptibility to colorectal cancer. Genet. Test. 2002, 6, 281-290. [CrossRef] [PubMed]

52. Niu, X.; Amendola, L.M.; Hart, R.; Bennette, C.S.; Heagerty, P.; Horike-Pyne, M.; Trinidad, S.B.; Rosenthal, E.A.; Comstock, B.; Nefcy, C.; et al. Clinical exome sequencing vs. usual care for hereditary colorectal cancer diagnosis: A pilot comparative effectiveness study. Contemp. Clin. Trials 2019, 84, 105820. [CrossRef] [PubMed]

53. Tiller, K.; Meiser, B.; Gaff, C.; Kirk, J.; Dudding, T.; Phillips, K.A.; Friedlander, M.; Tucker, K. A randomized controlled trial of a decision aid for women at increased risk of ovarian cancer. Med. Decis. Mak. 2006, 26, 360-372. [CrossRef] [PubMed]

54. Vogel, R.I.; Niendorf, K.; Petzel, S.; Lee, H.; Teoh, D.; Blaes, A.H.; Argenta, P.; Rivard, C.; Winterhoff, B.; Lee, H.Y.; et al. A patient-centered mobile health application to motivate use of genetic counseling among women with ovarian cancer: A pilot randomized controlled trial. Gynecol. Oncol. 2019, 153, 100-107. [CrossRef]

55. Zigmond, A.S.; Snaith, R.P. The hospital anxiety and depression scale. Acta Psychiatrica Scandinavica 1983, 67, 361-370. [CrossRef]

56. Speilberger, C.D.; Gorsuch, R.L.; Lushene, R.; Vagg, P.R.; Jacobs, G.A. State-Trait Anxiety Inventory for Adults; Mind Garden, Inc.: Redwood City, CA, USA, 1983.

57. Radloff, L.S. The Ces-D scale: A self-report depression scale for research in the general population. Appl. Psychol. Meas. 1977, 1, 385-401. [CrossRef]

58. Caswell-Jin, J.L.; Zimmer, A.D.; Stedden, W.; Kingham, K.E.; Zhou, A.Y.; Kurian, A.W. Cascade genetic testing of relatives for hereditary cancer risk: Results of an online initiative. J. Natl. Cancer Inst. 2019, 111, 95-98. [CrossRef]

59. Peshkin, B.N.; Ladd, M.K.; Isaacs, C.; Segal, H.; Jacobs, A.; Taylor, K.L.; Graves, K.D.; O’Neill, S.C.; Schwartz, M.D. The genetic education for men (Gem) trial: Development of web-based education for untested men in BRCA1/2-positive families. J. Cancer Educ. 2019, 11, 11.

60. Courtney, E.; Chok, A.K.; Ting Ang, Z.L.; Shaw, T.; Li, S.T.; Yuen, J.; Ngeow, J. Impact of free cancer predisposition cascade genetic testing on uptake in Singapore. NPJ Genom. Med. 2019, 4, 22. [CrossRef]

61. Forrest, L.E.; Burke, J.; Bacic, S.; Amor, D.J. Increased genetic counseling support improves communication of genetic information in families. Genet. Med. 2008, 10, 167-172. [CrossRef]

62. O’Neill, S.C.; Evans, C.; Hamilton, R.J.; Peshkin, B.N.; Isaacs, C.; Friedman, S.; Tercyak, K.P. Information and support needs of young women regarding breast cancer risk and genetic testing: Adapting effective interventions for a novel population. Fam. Cancer 2018, 17, 351-360. [CrossRef]

63. Evans, D.G.R.; Binchy, A.; Shenton, A.; Hopwood, P.; Craufurd, D. Comparison of proactive and usual approaches to offering predictive testing for BRCA1/2 mutations in unaffected relatives. Clin. Genet. 2009, 75, 124-132. [CrossRef] [PubMed]

64. Frey, M.K.; Kahn, R.M.; Chapman-Davis, E.; Tubito, F.; Pires, M.; Christos, P.; Anderson, S.; Mukherjee, S.; Jordan, B.; Blank, S.V.; et al. Prospective feasibility trial of a novel strategy of facilitated cascade genetic testing using telephone counseling. J. Clin. Oncol. 2020, 38, 1389-1397. [CrossRef] [PubMed]

65. Kardashian, A.; Fehniger, J.; Creasman, J.; Cheung, E.; Beattie, M.S. A pilot study of the sharing risk information tool (Sharit) for families with hereditary breast and ovarian cancer syndrome. Hered. Cancer Clin. Pract. 2012, 10, 4. [CrossRef] [PubMed]

66. Vrečar, I.; Hristovski, D.; Peterlin, B. Telegenetics: An update on availability and use of telemedicine in clinical genetics service. J. Med. Syst. 2017, 41, 21. [CrossRef]

67. Dheensa, S.; Fenwick, A.; Shkedi-Rafid, S.; Crawford, G.; Lucassen, A. Health-care professionals' responsibility to patients' relatives in genetic medicine: A systematic review and synthesis of empirical research. Genet. Med. 2016, 18, 290-301. [CrossRef]

68. Griffin, N.E.; Buchanan, T.R.; Smith, S.H.; Leon, A.A.; Meyer, M.F.; Liu, J.; Tabak, R.G.; Fuh, K.C.; Thaker, P.H.; Powell, M.A. Low rates of cascade genetic testing among families with hereditary gynecologic cancer: An opportunity to improve cancer prevention. Gynecol. Oncol. 2020, 156, 140-146. [CrossRef] [PubMed]

69. Menko, F.H.; ter Stege, J.A.; van der Kolk, L.E.; Jeanson, K.N.; Schats, W.; Ait Moha, D.; Bleiker, E.M.A. The uptake of presymptomatic genetic testing in hereditary breast-ovarian cancer and Lynch syndrome: A systematic review of the literature and implications for clinical practice. Fam. Cancer 2019, 18, 127-135. [CrossRef]

70. Unger, S.; Simond, E.; Davoine, E.; Katapodi, M.C. Dissemination of Genetic Information in Swiss Families with Lynch Syndrome: A Qualitative Exploratory Study. Clin. Oncol. Res. 2020, 2020, 1-5. [CrossRef]

71. Edwards, A.; Gray, J.; Clarke, A.; Dundon, J.; Elwyn, G.; Gaff, C.; Hood, K.; Iredale, R.; Sivell, S.; Shaw, C.; et al. Interventions to improve risk communication in clinical genetics: Systematic review. Patient Educ. Couns. 2008, 71, 4-25. [CrossRef]

72. Pedrazzani, C.; Caiata-Zufferey, M.; Kaiser-Grolimund, A.; Bürki, N.; Pagani, O.; Katapodi, M.C. CN115 communication of genetic information to family members in hereditary cancers and healthcare providers' role. Ann. Oncol. 2019, 30, v848. [CrossRef] 
73. Aceti, M.; Kaiser-Grolimund, A.; Pedrazzani, C.; Rudaz, M.; Baroutsou, V.; Bürki, N.; Chappuis, P.; Graffeo, R.; Monnerat, C.; Rabaglio, M.; et al. Bottom-up approach: Soliciting participants' input for developing a digital platform to support cancer coping and family communication about hereditary cancer risks in Switzerland and Korea. In Proceedings of the 5th Annual Meeting on Personalized Breast Cancer Treatments, Basel, Switzerland, 12-13 November 2020.

74. Hampel, H.; Frankel, W.L.; Martin, E.; Arnold, M.; Khanduja, K.; Kuebler, P.; Clendenning, M.; Sotamaa, K.; Prior, T.; Westman, J.A.; et al. Feasibility of screening for Lynch syndrome among patients with colorectal cancer. J. Clin. Oncol. 2008, 26, 5783-5788. [CrossRef] [PubMed]

75. Hampel, H.; Frankel, W.L.; Martin, E.; Arnold, M.; Khanduja, K.; Kuebler, P.; Nakagawa, H.; Sotamaa, K.; Prior, T.W.; Westman, J. Screening for the Lynch syndrome (hereditary nonpolyposis colorectal cancer). New Engl. J. Med. 2005, 352, 1851-1860. [CrossRef]

76. Win, A.K.; Jenkins, M.A.; Dowty, J.G.; Antoniou, A.C.; Lee, A.; Giles, G.G.; Buchanan, D.D.; Clendenning, M.; Rosty, C.; Ahnen, D.J. Prevalence and penetrance of major genes and polygenes for colorectal cancer. Cancer Epidemiol. Prev. Biomark. 2017, 26, 404-412. [CrossRef] [PubMed]

77. Haraldsdottir, S.; Rafnar, T.; Frankel, W.L.; Einarsdottir, S.; Sigurdsson, A.; Hampel, H.; Snaebjornsson, P.; Masson, G.; Weng, D.; Arngrimsson, R. Comprehensive population-wide analysis of Lynch syndrome in Iceland reveals founder mutations in Msh6 and Pms2. Nat. Commun. 2017, 8,1-11. [CrossRef]

78. Departments of Labor, Health and Human Services and the Treasury Genetic Information Nondiscrimination Act of 2008. Available online: https:/ / www.dol.gov/agencies/ebsa/laws-and-regulations/laws/gina (accessed on 27 January 2021).

79. Schwiter, R.; Kulchak Rahm, A.; Williams, J.L.; Curry Sturm, A. How can we reach at-risk relatives? Efforts to enhance communication and cascade testing uptake: A mini-review. Curr. Genet. Med. Rep. 2018, 6, 21-27. [CrossRef] 